\title{
CARTAN SUBALGEBRAS OF REGULAR EXTENSIONS OF VON NEUMANN ALGEBRAS
}

\author{
COLIN E. SUTHERLAND
}

(Received 20 December 1986)

Communicated by J. F. Price

\begin{abstract}
We analyse the structure of a regular extension $\mathscr{H} \rtimes_{\gamma, \nu} Q$ of a von Neumann algebra $\mathscr{M}$ by an action (modulo inner automorphisms) $\gamma$ of a discrete group $Q$, and a non-abelian 2-cycle $\nu$ for $\gamma$, under the assumption that the "action" $\gamma$ of $Q$ is cocycle conjugate to an "action" $\alpha$ which leaves globally invariant a Cartan subalgebra $\mathscr{E}$ of $\mathscr{M}$. We show that $\mathscr{M} \rtimes_{\gamma, \nu} Q$ is isomorphic with the algebra of the left regular projective representation of a certain discrete, non-principal groupoid $\mathscr{R} \vee Q$ determined by the action of $Q$ on the given Cartan subalgebra, where $\mathscr{R}$ is the Takesaki relation associated to the pair $(\mathscr{K}, \mathscr{C})$. We apply this description to give a decomposition of the regular representation of a group $G$ into irreducibles, where $G$ is a split extension of a type $I$ group $K$ by an abelian group $Q$, and work out the details of the author's earlier abstract Plancherel theorem in the case when $K$ is abelian.
\end{abstract}

1980 Mathematics subject classification (Amer. Math. Soc.) (1985 Revision): 46 L 10, 22 D 25.

\section{Introduction}

In an earlier paper, [15], this author has given a Plancherel formula for locally compact second countable groups $G$ whose regular representation $\rho^{G}$ is such that $\rho^{G}(G)^{\prime}$ contains a Cartan subalgebra in the sense of $[6]$-this includes all solvable or connected groups, since for such groups, $\rho^{G}(G)^{\prime \prime}$ is semifinite, [4], and injective, [2]. The formula is expressed in terms of data derived from irreducible representations of $G$ rather than factor representations occuring in the central decomposition of $\rho^{G}$ as has been the case with most "non-type I" Plancherel

(C) 1988 Australian Mathematical Society $0263-6115 / 88 \$ A 2.00+0.00$ 
formulae. In order to apply the Plancherel formula of [15], it is necessary to have at hand an explicit decomposition,

$$
\rho^{G}=\int_{X}^{\oplus} \rho_{x} d \mu(x)
$$

of $\rho^{G}$, with the diagonal subalgebra $\mathscr{D}$ of the decomposition being a Cartan subalgebra of $\rho^{G}(G)^{\prime}$; for $G$ solvable, connected, or discrete amenable, such a subalgebra exists and is unique up to automorphisms of $\rho^{G}(G)^{\prime}$.

This paper is an attempt, only partially successful, to construct such an algebra and decomposition when $G$ is an extension of a normal subgroup $K$ by a discrete group $Q$, and a suitable subalgebra of $\rho^{K}(K)^{\prime}$ is known. As is to be expected, this program is intimately related to that of [11], and its extension in [12] to the non-smooth case, which seeks to describe all of the irreducible representations of $G$ in terms of those in $K$ and the action of $G$ on this space. Our goal is more limited - we seek only sufficiently many irreducibles of $G$ to decompose $\rho^{G}$. Our point of view is also somewhat different from that of [11] or [12]; rather than work with the space $\hat{K}$ of equivalence classes of irreducible representation of $K$, we work with (a part of) the category $\operatorname{Irr}(K)$ of concrete irreducible representations of $K$, with intertwiners as morphisms, together with the natural action of $G$.

In order to perform our analysis, we need to make some assumptions on the action of $G$ on $\mathscr{M}=\rho^{K}(K)^{\prime \prime}$; roughly speaking we need to assume the existence of an action $\alpha$ of $G$ on $\mathscr{K}$ conjugate to the natural action, and which leaves some Cartan subalgebra $\mathscr{C}$ of $\mathscr{K}$ globally invariant - the precise requirement, given in Assumption 3.1, is somewhat weaker than this, and applies whenever $G$ is discrete amenable, or $Q$ is discrete amenable, $1 \rightarrow K \rightarrow G \rightarrow Q \rightarrow 1$ is split, and $\rho^{K}(K)^{\prime \prime}$ is injective and semifinite, using results of [17].

For us, the main virtue of working with decompositions over Cartan subalgebras is that such a decomposition leads to a description of the algebra in question as an algebra of "coherent sections" (or "random operator fields" in the terminology of [1]). More precisely if $\mathscr{K}$ is a von Neumann algebra in standard form on a Hilbert space $\mathscr{H}$ and $\mathscr{D} \subseteq \mathscr{M}^{\prime}$ is any maximal abelian subalgebra, and if $\mathscr{A} \subseteq \mathscr{M}$ is any $\sigma$-weakly dense separable $C^{*}$-subalgebra, then the decomposition of the self-representation $\iota \mathscr{A}$ of $\mathscr{A}$ over $\mathscr{D}$,

$$
\iota_{\mathscr{A}}=\int_{X}^{\oplus} \pi_{x} d \mu(x)
$$

gives rise to an equivalence relation $\mathscr{R}$ on $X$ defined by $(x, y) \in \mathscr{R}$ if and only if $\pi_{x} \stackrel{\sim}{\sim} \pi_{y}$, where $\stackrel{\sim}{\sim}$ denotes unitary equivalence. According to [18], $\mathscr{R}$ is (effectively) independent of the choice of $\mathscr{A}$. In addition, by [13], if we choose unitaries $v(x, y)$ such that $\operatorname{Ad} v(x, y) \circ \pi_{y}=\pi_{x}$ for $(x, y) \in \mathscr{R}$, then $v(x, y) v(y, z)=$ $f(x, y, z) v(x, z)$ for some 2-cocycle $f \in Z^{2}(\mathscr{R}, \mathbf{T})$ on $\mathscr{R}$. The results of [13] also 
tell us that if $\mathscr{D}$ is a Cartan subalgebra, $\mathscr{R}$ is a countable non-singular equivalence relation as defined in [6], and the Hilbert space of $\pi_{x}$ may be identified with $l^{2}(\mathscr{R}(x)=\{y:(x, y) \in \mathscr{R}\})$; furthermore we may take $v(x, y)=L^{f}(x, y)$ where $\left(L^{f}(x, y) \xi\right)(z)=f(z, x, y) \xi(z)$, and the algebra $\mathscr{C}$ is unitarily equivalent to the algebra $\Gamma_{\mu}^{\infty}\left(\operatorname{Ad} L^{f}\right)$ of essentially bounded measurable operator fields $x \in X \rightarrow T(x) \in B\left(l^{2}(\mathscr{R}(x))\right)$ which satisfy $L^{f}(x, y) T(y)=T(x) L^{f}(x, y)$ a.e. on $\mathscr{R}$ (where we identify fields which agree a.e.). It is this description of $\mathscr{M}$ which allows our analysis to proceed.

The organization of the paper is as follows; $\S 2$ is preparatory, and shows how to synthesise a measured groupoid $(\mathscr{R} \vee Q, \mu)$ out of measured discrete equivalence relation $(\mathscr{R}, \mu)$ and a discrete group $Q$ acting, modulo inner automorphisms, on $(\mathscr{R}, \mu)$. We also provide a description of the cohomology group $H_{\mu}^{2}(\mathscr{R} \vee Q ; T)$ needed in the subsequent analysis. In $\S 3$, we analyze regular extensions, [14], $\mathscr{K} \times_{\alpha, \omega} Q$ of a von-Neumann algebra $\mathscr{K}$ by an action $\alpha$ (modulo inner automorphisms) of $Q$ and a (non-abelian) 2-cocycle $\omega$, under the assumption that $\alpha$ admits an invariant Cartan subalgebra $\mathscr{C}$. Using the results of [6, 13] described above (see also [9]), we know that in this case $\mathscr{M}=\Gamma_{\mu}^{\infty}\left(\operatorname{Ad} L^{f}\right)$; we prove, in Theorem 3.8 that $\mathscr{M} \rtimes_{\alpha, \omega} Q=\Gamma_{\mu}^{\infty}\left(\operatorname{Ad} L^{\hat{f}}\right)$ for some explicitly determined $\hat{f} \in H_{\mu}^{2}(R \vee Q, \mathbf{T})$.

The groupoid $\mathscr{R} \vee Q$ is not in general principal, that is, not an equivalence relation. Indeed, it has isotropy groups $Q_{x}=\left\{s \in Q: \pi_{x} \circ \alpha_{s} \stackrel{u}{\sim} \pi_{x}\right\}$ where $\left\{\pi_{x}\right\}$ are as above (the decomposition being over $\mathscr{D}=J \mathscr{C} J$, where $J$ is a modular involution for $\mathscr{K}$. The algebra $\Gamma_{\mu}^{\mu}\left(\operatorname{Ad} L^{\hat{f}}\right)$ may then be described as the algebra of bounded measurable operator fields $x \in X \rightarrow T(x) \in B\left(\mathscr{H}_{x} \otimes l^{2}\left(Q_{x}\right)\right)$, where $\mathscr{H}_{x}$ is the Hilbert space of $\pi_{x}$, which commute with $1 \otimes \lambda^{c_{x}}\left(Q_{x}\right)$, and which are "coherent" with respect to a natural action of the underlying equivalence relation $\mathscr{S}$ of $\mathscr{R} \vee Q$; here $\lambda^{c_{x}}$ is the left regular projective representation of $Q_{x}$ for a certain multiplier $c_{x}$ of $Q_{x}$ derived from $\hat{f}$. This is accomplished in $\S 4$. In $\S 5$, we apply our results to exact sequences $1 \rightarrow K \rightarrow G \rightarrow Q \rightarrow 1$ of locally compact second countable groups, with $Q$ discrete, and for which the associated "action" of $Q$ on $\rho^{K}(K)^{\prime \prime}$ satisfies Assumption 3.1. Our conclusion, Theorem 5.3, is that there is a decomposition $\rho^{K}=\int_{X}^{\otimes} \rho_{x} d \mu(x)$ of $\rho^{K}$ into irreducibles such that if $G_{x}=\left\{g \in G: \rho_{x} \circ \operatorname{Ad} g \stackrel{\sim}{\sim} \rho_{x}\right\}$ and $\psi_{x}=\operatorname{Ind}_{K}^{G_{x}} \rho_{x}$ then

(i) if $\psi_{x}=\int_{U_{x}}^{\oplus} \psi_{x, u} d \mu_{x}(u)$ is any decomposition of $\psi_{x}$ into irreducibles, $\int_{U_{x}}^{\oplus} \operatorname{Ind}_{G_{x}}^{G} \psi_{x, u} d \mu_{x}(u)$ is a decomposition of $\hat{\rho}_{x}=\operatorname{Ind}_{K}^{G} \rho_{x}$ into irreducibles;

(ii) all irreducible decompositions of $\hat{\rho}_{x}$ arize in the manner described in i).

The commutant of $\psi_{x}$ is identified explicitly as being isomorphic with $\lambda^{c_{x}}\left(Q_{x}\right)^{\prime \prime}$, where $Q_{x}=G_{x} / K$, and $c_{x}$ is identified with the (Mackey) multiplier naturally attached to the representation $\rho_{x}$ of $K$. Thus in specific instances we can, in principal, find irreducible decompositions of $\rho^{G} ; \S 5$ also presents some 
(non-type I) examples where this procedure can be made explicit, and computes the data necessary for application of the Plancherel Theorem of [15].

As stated earlier, the final goals of the program are only partially met; even with abelian groups $Q$, the algebras $\lambda^{c_{x}}\left(Q_{x}\right)^{\prime \prime}$ may fail to be type I, although they are always finite, and injective if $Q$ is merely amenable. Thus there is no known constructive procedure for constructing irreducible decompositions of the representations $\psi_{x}$ above. The machinery needed to obtain the results presented here is quite elaborate, and the results themselves a little cumbersome; the author feels that while it may well be possible to generalize the results to include nondiscrete groups $Q$, it may be preferable to instead concentrate on specific (families of) discrete groups, and on constructions of concrete (families of) irreducible representations of them, rather than to pursue the "normal subgroup" approach used here. The results of this paper are intended more as guidelines than as models.

\section{Extensions of equivalence relations and their cohomology}

Throughout, $(\mathscr{R}, \mu)$ will denote an (orbitally) discrete measured equivalence relation as in [6], $r$ and $s$ denote the range and source maps respectively, $X=$ $\mathscr{R}^{(0)}$ the space of units, and $\mathscr{R}^{(2)}$ the domain of the composition; $\delta(x, y)=$ $d \mu \circ r / d \mu \circ s(x, y)$ denotes the modular homomorphism. $H$ will denote a discrete group.

DEFINITION 2.1. An action of $H$ on $(\mathscr{R}, \mu)$ is a Borel action $(x, h) \in X \times$ $H \rightarrow x h \in X$ of $H$ on $X$ with

(i) $\mu(E h)=0$ if $E \subseteq X$ and $\mu(E)=0$;

(ii) $(x, y) \in \mathscr{R}$ if and only if $(x h, y h) \in \mathscr{R}$ for all $h \in H$.

Given an action of $H$ on $(\mathscr{R}, \mu)$, we may construct a standard Borel groupoid $\mathscr{G}=\mathscr{R} \times{ }_{s} H$ by declaring

$$
\begin{aligned}
\mathscr{G}^{(2)}=\{(x, y, h),(y h, z h, g)) \in(\mathscr{R} \times H) \times(\mathscr{R} \times H): & \\
(x, y) & \in \mathscr{R} \text { and }(y, z) \in \mathscr{R}\},
\end{aligned}
$$

and $(x, y, h)(y h, z h, g)=(x, z, h g)$. We have $r(x, y, h)=x$ and $s(x, y, h)=y h$ in $\mathscr{R} \times{ }_{s} H$, and $(x, y, h)^{-1}=\left(y h, x h, h^{-1}\right)$. See $[1,6,12]$ for further discussion of groupoids.

Assume now that $H$ acts on $(\mathscr{R}, \mu)$ and that $N$ is a fixed normal subgroup of $H$ with the property that $(x n, x) \in \mathscr{R}$ for all $n \in N$; in this case we say that $N$ acts via inner automorphisms. Define an equivalence relation $\sim_{N}$ on $\mathscr{R} \times{ }_{s} H$ by $(x, y, h) \sim_{N}\left(x_{1}, y_{1}, h_{1}\right)$ if and only if $x=x_{1}$ and $\left(y_{1}, h_{1}\right)=\left(y n, n^{-1} h\right)$ for some 
$n \in N$. Note that if $\left((x, y, h),\left(x^{\prime}, y^{\prime}, h^{\prime}\right)\right) \in\left(\mathscr{R} \times \times_{s} H\right)^{(2)}$, and if $\left(x_{1}, y_{1}, h_{1}\right) \sim_{N}$ $(x, y, h)$ and $\left(x_{1}^{\prime}, y_{1}^{\prime}, h_{1}^{\prime}\right) \sim_{N}\left(x^{\prime}, y^{\prime}, h^{\prime}\right)$, then $\left(\left(x_{1}, y_{1}, h_{1}\right),\left(x_{1}^{\prime}, y_{1}^{\prime}, h_{1}^{\prime}\right)\right) \in \mathscr{R} \times_{s}$ $H)^{(2)}$ and $(x, y, h)\left(x^{\prime}, y^{\prime}, h^{\prime}\right) \sim_{N}\left(x_{1}, y_{1}, h_{1}\right)\left(x_{1}^{\prime}, y_{1}^{\prime}, h_{1}^{\prime}\right)$. Thus the quotient $\mathscr{R} \times_{s}$ $H / \sim_{N}$ becomes a standard Borel groupoid which we denote by $\mathscr{R} \vee Q$, where $Q=H / N$. It is largely groupoids of this form which will be of interest in the remainder of the paper.

There is an alternate description of $\mathscr{R} \vee Q$ which will be useful also. If $s \in Q \rightarrow h_{s} \in H$ is a section for the map $p: H \rightarrow Q$, and $[x, y, h]$ denotes the class of $(x, y, h) \in \mathscr{R} \times{ }_{s} H$ in $\mathscr{R} \vee Q$, the map $(x, y, s) \in \mathscr{R} \times Q \rightarrow\left[x, y, h_{s}\right] \in \mathscr{R} \vee Q$ provides a Borel isomorphism of the underlying Borel space of $\mathscr{R} \vee Q$ with $\mathscr{R} \times Q$. Transferring the groupoid structure on $\mathscr{R} \vee Q$ to $\mathscr{R} \times Q$, we obtain a product on $\mathscr{R} \times Q$ given by

$$
(x, y, s)\left(y h_{s}, z h_{s}, t\right)=\left(x, z h_{s} h_{t} h_{s t}^{-1}, s t\right) .
$$

Thus $\mathscr{R} \vee Q$ is to be viewed as a general extension of $\mathscr{R}$ by $Q$; note also that if $1 \rightarrow N \rightarrow H \rightarrow Q \rightarrow 1$ is split and $s \rightarrow h_{s}$ is a homomorphism, then $(x, s) \rightarrow x h_{s}$ provides an action of $Q$ on $(\mathscr{R}, \mu)$ and $\mathscr{R} \vee Q$ coincides with $\mathscr{R} \times{ }_{s} Q$.

For subsequent applications, we will need a description of the cohomology groups $H_{\mu}^{2}(\mathscr{R} \vee Q ; A)$ and $H^{2}\left(\mathscr{R} \times_{s} H ; A\right)$, where $A$ is a fixed abelian group, typically the circle group $T$. Since the groupoids $\mathscr{R} \times{ }_{s} H, \mathscr{R} \vee Q$ may fail to be principal, these cohomology groups do not fall under the discussion of [6], so we give a brief description of their development.

For $\mathscr{G}=\mathscr{R} \vee Q$ or $\mathscr{R} \times{ }_{s} H$, let $\mathscr{C}^{n}(\mathscr{G})$ denote the space of equivalence classes modulo equality a.e. on $\mathscr{G}^{(n)}$, of Borel functions $f: \mathscr{G}^{(n)} \rightarrow A$ with

$$
f\left(\gamma_{1}, \gamma_{2}, \ldots, \gamma_{n}\right)=0 \text { if any } \gamma_{j} \text { is a unit. }
$$

Define successively

(a) $\partial_{n}: \mathscr{C}^{n}(\mathscr{G}) \rightarrow \mathscr{C}^{n+1}(\mathscr{G})$ by

$$
\begin{aligned}
\left(\partial_{n} f\right)\left(\gamma_{1}, \ldots, \gamma_{n+1}\right)= & f\left(\gamma_{2}, \gamma_{3}, \ldots, \gamma_{n+1}\right)+(-1)^{n+1} f\left(\gamma_{1}, \gamma_{2}, \ldots, \gamma_{n}\right) \\
& +\sum_{j=1}^{n}(-1)^{j} f\left(\gamma_{1}, \ldots, \gamma_{j} \gamma_{j+1}, \ldots, \gamma_{n}\right),
\end{aligned}
$$

(b) $Z_{\mu}^{n}(\mathscr{G}, A)=\operatorname{ker} \partial_{n} ; B_{\mu}^{n}(\mathscr{G}, A)=\operatorname{Image} \partial_{n-1}$, and

$$
H_{\mu}^{n}(\mathscr{G}, A)=Z_{\mu}^{n}(\mathscr{G}, A) / B^{n}(\mathscr{G}, A) \text {. }
$$

Note $H_{\mu}^{n}(\mathscr{G}, A)$ is well defined as $\partial_{n+1} \circ \partial_{n}=0$, and that our definition agrees with that of $[6]$ in case is $\mathscr{G}$ an equivalence relation.

The proper explanation for the analysis of $H_{\mu}^{2}\left(\mathscr{R} \times{ }_{s} H ; A\right)$ which follows undoubtedly lies in a spectral sequence for $H_{\mu}^{n}(\mathscr{G} ; A)$ with the $E^{2}$-term $H_{\mu}^{p}\left(H ; H_{\mu}^{q}(\mathscr{R} ; A)\right)$; since the applications we have in mind do not seem to necessitate such machinery, we will not attempt its development here. We will assume throughout the discussion that the coefficient group $A$ is divisible. 
LEMMA 2.1. Each element of $Z_{\mu}^{2}\left(\mathscr{R} \times{ }_{s} H ; A\right)$ is cohomologous to an element $F \in Z_{\mu}^{2}\left(\mathscr{R} \times{ }_{s} H ; A\right)$ which satisfies

(a) $F\left(\gamma, \gamma^{-1}\right)=0$ for $\gamma \in \mathscr{R} \times{ }_{s} H$;

(b) $F((x, x, h),(x h, y h, e))=0$ for $(x, y) \in \mathscr{R}, h \in H$.

Proof. (a) This follows precisely as in [6, Lemma 7.6] since $A$ is (assumed) divisible.

(b) If $F_{1} \in Z_{\mu}^{2}\left(\mathscr{R} \times{ }_{s} H ; A\right)$ is arbitrary, define

$$
a(x, y, h)=F_{1}((x, x, h),(x h, y h, e)) .
$$

Then if $F=F_{1} \partial a$,

$$
F((x, x, h),(x h, y h, e))=F_{1}((x, x, h),(x h, y h, e))-a(x, y, h)=0,
$$

as $a(x, x, h)=a(x h, y h, e)=0$. Also, if $F_{1}$ satisfies the normalization in $\left.a\right)$, so does $F$.

THEOREM 2.2. Let $F \in Z_{\mu}^{2}\left(\mathscr{R} \times{ }_{s} H ; A\right)$ be normalized as in Lemma 2.1, and define for $((x, y),(y, z)) \in \mathscr{R}^{(2)}$ and $g, h, k \in H$,

$$
\begin{aligned}
f(x, y, z) & =F((x, y, e),(y, z, e)), \\
a_{h}(x, y) & =-F((x, y, e),(y, y, h)), \\
c_{g, h}(x) & =F((x, x, g),(x g, x g, h)) \\
A(g, x, y, z) & =a_{g}(x, y)+a_{g}(y, z)-a_{g}(x, z)+f(x, y, z)-f(x g, y g, z g), \\
B(g, h, x, y) & =a_{h}(x g, y g)+a_{g}(x, y)-a_{g h}(x, y)+c_{g, h}(y)-c_{g, h}(x), \\
C(g, h, k, x) & =c_{h, k}(x g)+c_{g, h k}(x)-c_{g h, k}(x)-c_{g, h}(x) .
\end{aligned}
$$

Then (i) $f \in Z_{\mu}^{2}(\mathscr{R}, A)$ and

$$
F((x, y, g),(y g, z g, h))=f(x g h, y g h, z g h)+c_{g, h}(x)-a_{h}(x g, y g) ;
$$

(ii) for all $x, y, z, g, h$ and $k$,

$$
\begin{gathered}
A(g, x, y, z)=B(g, h, x, y)=C(g, h, k, z)=0, \text { and } \\
a_{h}(x, y)+a_{h}(y, x)=a_{g}(z, z)=0
\end{gathered}
$$

(iii) if $f \in Z_{\mu}^{2}(\mathscr{R}, A)$ is given, and we are also given Borel functions $a_{h}: \mathscr{R} \rightarrow$ $A, c_{g, h}: X \rightarrow A$ satisfying the identities in (ii), then the formula in (i) defines an element of $Z_{\mu}^{2}\left(\mathscr{R} \times{ }_{s} H ; A\right)$. 
ProOF. Let $F \in Z_{\mu}^{2}\left(\mathscr{R} \times{ }_{s} H ; A\right)$ be given, and define $f, a_{h}, c_{g, h}$ as above. Clearly $f \in Z_{\mu}^{2}(\mathscr{R}, A)$, and we calculate

$$
\begin{aligned}
F((x, y, g),(y g, z g, h))= & f((x, x, g)(x g, y g, e),(y g, z g, h)) \\
= & F((x, x, g),(x g, x g, h)(x g h, z g h, e)) \\
& +F((x g, y g, e),(y g, y g, h)(y g h, z g h, e)) \\
= & c_{g, h}(x)-a_{h}(x g, y g)+f(x g h, y g h, z g h),
\end{aligned}
$$

where we have used the normalization of $F$ in Lemma 2.1(b) and the cocycle identity for $F$ with the indicated variables at each stage. Thus (i) is verified.

It is clear from the definition that $a_{h}(x, x)=1$; the relation $a_{h}(x, y)+$ $a_{h}(y, x)=0$ follows from Lemma 2.1(a).

To establish the remainder of (ii), and (iii), suppose $f \in Z_{\mu}^{2}(\mathscr{R}, A)$ is given, and we are given arbitrary Borel functions $a_{h}: \mathscr{R} \rightarrow A, c_{g, h}: X \rightarrow A$ with $a_{h}(x, x)=a_{h}(x, y)+a_{h}(y, x)=0$. Define $F:\left(\mathscr{R} \times_{s} H\right)^{(2)} \rightarrow A$ by

$$
F\left((x, y, g),(y g, z g, h)=f(x g h, y g h, z g h)+c_{g, h}(x)-a_{h}(x g, y g) .\right.
$$

Using the cocycle identity for $f$ with the variables $x g h k, y g h k, z g h k$ and $w g h k$, we find

$$
\begin{aligned}
& (\partial F)((x, y, g),(y g, z g, h),(z g h, w g h, k)) \\
& \quad=B(h, k, x g, y g)+C(g, h, k, x)-A(k, x g h, y g h, z g h) .
\end{aligned}
$$

Thus, if the identities in (ii) are satisfied, $F \in Z_{\mu}^{2}\left(\mathscr{R} \times{ }_{s} H ; A\right)$. Conversely, if $F \in Z_{\mu}^{2}\left(\mathscr{R} \times{ }_{s} H ; A\right)$, and $a_{h}, c_{g, h}$ are as defined in (ii) then, using (i) we have

$$
B(h, k, x g, y g)+C(g, h, k, x)-A(k, x g h, y g h, z g h)=0 .
$$

In particular, $A(k, x g h, y g h, z g h)$ is independent of $z$, and taking $z=y$, we obtain $A=0$. Similarly, $B(h, k, x g, y g)$ is independent of $y$ and hence zero, and $C=0$.

REMARK 2.3. The concluding argument in the above proof depends crucially on the diagonal in $X \times X$ having positive $\mathscr{R}$-measure. In addition if $H$ is allowed to become locally compact and second countable, $\mathscr{R} \times\{e\}$ may have measure zero in $\mathscr{R} \times{ }_{s} H$ so that, for example, $f$ is no longer well defined. Thus any extension to more general groupoids and groups of Theorem 2.2 is fraught with measure-theoretic difficulties.

REMARK 2.4. The conditions $A=0, B=0, C=0$ in Theorem 2.2(ii) may be rephrased by saying $f \in H_{\mu}^{2}(\mathscr{R} ; A)^{H}$ for the natural action of $H$ on $H_{\mu}^{2}(\mathscr{R}, A)$, $g \rightarrow a_{g}$ defines an element of $Z_{\mu}^{1}\left(H ; H_{\mu}^{1}(\mathscr{R}, A)\right)$, and $(g, h) \rightarrow c_{g, h}$ defines an element of $Z^{2}\left(H ; H_{\mu}^{0}(\mathscr{R} ; A)\right)$. We thus see all the terms expected from spectral sequences appearing. 
We now turn to a discussion of $H_{\mu}^{2}(\mathscr{R} \vee Q ; A)$ where $Q=H / N$, and $N$ acts via inner automorphisms. Let $p: \mathscr{R} \times{ }_{s} H \rightarrow \mathscr{R} \vee Q$ be the quotient map, and define $p_{*}: Z_{\mu}^{2}(\mathscr{R} \vee Q ; A) \rightarrow Z_{\mu}^{2}\left(\mathscr{R} \times_{s} H ; A\right)$ by $p_{*} \hat{f}=\hat{f} \circ(p \times p)$ for $\hat{f} \in Z_{\mu}^{2}(\mathscr{R} \vee Q ; A)$. The range of $p_{*}$ evidently consists of those 2 -cycles on $\mathscr{R} \times{ }_{s} H$ which are invariant under the (left) action $((x, y, g),(y g, z g, h)) \rightarrow\left(\left(x, y n^{-1}, n g\right),\left(y g, z g m^{-1}, m h\right)\right)$ of $N \times N$ on $\left(\mathscr{R} \times{ }_{s} H\right)^{(2)}$. It is straight-forward to describe this range in terms of the description of 2-cycles offered in Theorem 2.2; we leave this to the reader.

Proposition 2.5. $p_{*}$ determines an injection of $H_{\mu}^{2}(\mathscr{R} \vee Q ; A)$ into $H_{\mu}^{2}\left(\mathscr{R} \times_{s} H ; A\right)$.

Proof. Suppose $\hat{f}_{1}, \hat{f}_{2} \in Z_{\mu}^{2}(\mathscr{R} \vee Q, A)$, and $p_{*} \hat{f}_{1}=F_{1}, p_{*} \hat{f}_{2}=F_{2}$ with $F_{1}=F_{2}+\partial d$. Since $F_{1}, F_{2}$ are invariant under the action of $N \times\{e\}$ we obtain

$$
d(x, y, g)-d\left(x, y n^{-1}, n g\right)=d(x, z, g h)-d\left(x, z n^{-1}, n g h\right) .
$$

Thus $d(x, y, g)-d\left(x, y n^{-1}, n g\right)$ is independent of $g$ and of $y$ (with $(x, y) \in \mathscr{R}$ ). Similarly, using invariance under $\{e\} \times N$,

$$
d(y g, z g, h)-d\left(y g, z g m^{-1}, m h\right)=d(x, z, g h)-d\left(x, z g m^{-1} g^{-1}, g m h\right),
$$

from which it follows that $d(x, y, g)-d\left(x, y n^{-1}, n g\right)=b_{x}(n)$ depends only on $n$ and the class of $x$ in $\mathscr{R}$. Note that $b_{x}$ is a character on $N$, and that, from the second of the above invariance conditions,

$$
b_{y g}(m)=b_{x}\left(g m g^{-1}\right) \quad \text { for } g \in H,(x, y) \in \mathscr{R} .
$$

Thus if $s \in Q \rightarrow h_{s} \in H$ is a section, and

$$
d^{\prime}\left(x, y n^{-1}, n h_{s}\right)=b_{x}(n)+d\left(x, y n^{-1}, n h_{s}\right),
$$

$d^{\prime}$ is $N$-invariant and $\partial d=\partial d^{\prime}$. Since $d^{\prime}=\hat{d} \circ p$ for some $\hat{d}$ defined on $\mathscr{R} \vee Q$, $\hat{f}_{1}=\hat{f}_{2}+\partial \hat{d}$ as required.

We note that it is possible to analyze $Z_{\mu}^{2}(\mathscr{R} \vee Q ; A)$ along the lines of Theorem 2.2. However, this is algebraically very messy indeed, and omitted for lack of elegance and application - the reader need only examine the 2-cycle on $\mathscr{R} \vee Q$ described in Theorem 3.8 to appreciate the complexity of the situation.

\section{Regular extensions of algebras of sections}

Throughout this section, $\mathscr{M}$ is a von Neumann algebra acting on a separable Hilbert space $\mathscr{H}, U(\mathscr{K})$ is the unitary group of $\mathscr{M}$, and $Q$ is a discrete group. We assume we are given maps $s \in Q \rightarrow \gamma_{s} \in \operatorname{Aut}(\mathscr{M})$ and $(s, t) \in Q \times Q \rightarrow$ $\nu(s, t) \in U(\mathscr{M})$ satisfying

$$
\gamma_{s} \circ \gamma_{t}=\operatorname{Ad} \nu(s, t) \circ \gamma_{s t} \text { on } Q \times Q
$$




$$
\gamma_{r}(\nu(s, t)) \nu(r, s t)=\nu(r, s) \nu(r s, t) \text { on } Q \times Q \times Q .
$$

We will be interested in the structure of the regular extension $\varkappa_{\gamma, \nu} Q$ as defined in [14], under a certain assumption concerning the "action" of $Q$. This assumption is rather technical in nature and is easiest to state after the following construction has been made.

Let $U_{0} \subseteq U(\mathscr{M})$ be the smallest subgroup which contains $\{\nu(r, s): r, s \in Q\}$ and is invariant under $\left\{\gamma_{s}: s \in Q\right\}$; endow $H=U_{0} \times Q$ with product

$$
(u, r)(v, s)=\left(u \gamma_{r}(v) \nu(r, s), r s\right),
$$

so that $H$ is a discrete group also. Note there is a homomorphism $\beta: h \in H \rightarrow$ $\beta_{h} \in \operatorname{Aut}(\mathscr{M})$ given by

$$
\beta_{(u, r)}=\operatorname{Ad} u \circ \gamma_{r}
$$

Assumption 3.1. $\mathscr{M}$ is in standard form, and the action $\beta$ of $H$ on $\mathscr{M}$ is cocycle conjugate to an action $\alpha$ of $H$ on $\mathscr{K}$ for which there is a Cartan subalgebra $\mathscr{C} \subseteq \mathscr{M}$ invariant under $\left\{\alpha_{h}: h \in H\right\}$.

Here, standard form is as defined in [8], cocycle conjugacy is as discussed in (for example) [10], and a Cartan subalgebra is as defined in [6]. Despite its somewhat peculiar appearance, Assumption 3.1 is met in many non-trivial cases - for example, using $[17, \S 5]$, it holds whenever $\mathscr{M}$ is semifinite and injective, and $H$ can be chosen to be amenable. Note that we may take $\alpha_{h}=\operatorname{Ad} u_{h} \circ \beta_{h}$ for some 1-cocycle $u \in Z_{\beta}^{1}(H, U(\mathscr{M}))$, since if $\theta \circ \operatorname{Ad} u_{h} \circ \beta_{h} \circ \theta^{-1}$ admits an invariant Cartan subalgebra, so does $\operatorname{Ad} u_{h} \circ \beta_{h}$.

Note that if we write $\alpha_{s}=\alpha_{(1, s)}, \beta_{s}=\beta_{(1, s)}=\gamma_{s}$ and $u_{s}=u_{(1, s)}$ for $s \in Q$, then with $\omega(s, t)=u_{s} \beta_{s}\left(u_{t}\right) \nu(s, t) u_{s t}^{*}$,

$$
\begin{gathered}
\alpha_{s} \circ \alpha_{t}=\operatorname{Ad} \omega(s, t) \circ \alpha_{s t} \text { on } Q \times Q, \\
\alpha_{r}(\omega(s, t)) \omega(r, s t)=\omega(r, s) \omega(r s, t) \quad \text { on } Q \times Q \times Q .
\end{gathered}
$$

We take the generators of $\mathscr{M} \rtimes_{\alpha, \omega} Q$ on $l^{2}(Q ; \mathscr{H})$ to be

$$
\begin{aligned}
& \left(\pi^{\alpha}(x) \xi\right)(s)=\alpha_{s}(x) \xi(s), \quad x \in \mathscr{M}, \\
& \left(\rho^{\omega}(t) \xi\right)(s)=\omega(s, t) \xi(s t), \quad t \in Q,
\end{aligned}
$$

and similarly for $\rtimes_{\gamma, \nu} Q$. Note these are unitary transforms of the generators used in [14] by the unitary $(j \xi)(s)=\xi\left(s^{-1}\right)$.

LEMMA 3.2. (a) The map

$$
\begin{aligned}
\pi^{\gamma}(x) & \rightarrow \pi^{\alpha}(x), \quad x \in \mathscr{K}, \\
\rho^{\nu}(s) & \rightarrow \pi^{\alpha}\left(u_{s}^{*}\right) \rho^{\omega}(s), \quad s \in Q,
\end{aligned}
$$

extends to a unitary equivalence of $\mathscr{M} \rtimes_{\gamma, \nu} Q$ with $\mathscr{M} \rtimes_{\alpha, \omega} Q$. 
(b) If $\mathscr{W}$ is in standard form, and $\left\{a_{s}: s \in Q\right\}$ are the canonical unitaries (as in [8]) implementing $\left\{\alpha_{s}: s \in Q\right\}$, then the commutant of $\mathscr{M} \rtimes_{\alpha, \omega} Q$ is generated by

$$
\begin{aligned}
(\bar{y} \xi)(s) & =y \xi(s), \quad y \in \mathscr{M}^{\prime}, \\
\left(\lambda^{\omega}(t) \xi\right)(s) & =a_{t-1}^{*} \omega\left(t^{-1}, s\right) \xi\left(t^{-1} s\right), \quad t \in Q .
\end{aligned}
$$

ProOF. (a) The unitary $U$ on $l^{2}(Q, \mathscr{H})$ defined by $(U \xi)(s)=u_{s} \xi(s)$ implements the indicated isomorphism.

(b) It is routine to check that $\bar{y}$ and $\lambda^{\omega}(t)$ lie in $\left(\mathscr{M} \rtimes_{\alpha, \omega} Q\right)^{\prime}$ for $y \in \mathscr{M}^{\prime}$ and $t \in Q$. Conversely, if $z \in B\left(l^{2}(Q ; \mathscr{H})\right)$ commutes with $\left\{\bar{y}: y \in \mathscr{M}^{\prime}\right\}$, then $z \in \mathscr{M} \times B\left(l^{2}(Q)\right)$, so we can write $z=\left(z_{r, s}\right)$ as an $\mathscr{M}$-valued matrix. If $z$ also commutes with $\lambda^{\omega}(t)$ for all $t$, we obtain

$$
z_{r s, r t}=\omega(r, s)^{*} \alpha_{r}\left(z_{s, t}\right) \omega(r, t)
$$

for all $r, s, t \in Q$. From this, one now obtains

$$
z=\sum_{r} \rho^{\omega}(r)^{*} \pi^{\alpha}\left(z_{r, e}\right) \in \mathscr{M} \rtimes_{\alpha, \omega} Q
$$

We now proceed to examine $\mathscr{M} \rtimes_{\alpha, \omega} Q$. The following auxiliary construction will be useful. Note that if $N=U_{0} \times\{e\} \subseteq H, N$ is normal in $H$ with $H / N=Q$; further, there is a unitary representation $n \in N \rightarrow t_{n} \in U(\mathscr{M})$ with $\alpha_{n}=\operatorname{Ad} t_{n}$ on $N$. Let $\mathscr{A}$ be any separable, $\sigma$-weakly dense ${ }^{*}$-subalgebra of $\mathscr{A}$ which is $\alpha$-invariant, and contains both $\left\{t_{n}: n \in N\right\}$ and $\left\{u_{h}: h \in H\right\}$; the system $(\mathscr{A}, H, \alpha, t)$ is now a twisted covariant system in the sense of [7].

Let $J$ be a modular involution for $\mathscr{K}$, and set $\mathscr{D}=J \mathscr{C} J \subseteq \mathscr{M}^{\prime}$. Let

$$
\left\{\iota_{\mathscr{A}}, \mathscr{H}\right\}=\int_{X}^{\oplus}\left\{\pi_{x}, \mathscr{H}_{x}\right\} d \mu(x)
$$

be the decomposition of the identity representation $\iota \mathscr{A}$ of $\mathscr{A}$ with diagonal algebra $\mathscr{D}$. Let $\mathscr{R}$ be the Takesaki relation on $X,[18]$, so that $(x, y) \in \mathscr{R}$ if and only if $\pi_{x} \stackrel{u}{\sim} \pi_{y}$ where $\stackrel{u}{\sim}$ denotes unitary equivalence; let $\sigma(x, y)$ denote the associated (automorphic) representation of $\mathscr{R},[13]$, determined by the equation $\sigma(x, y) \circ \pi_{y}=\pi_{x}$, and choose a unitary-valued Borel map $(x, y) \in \mathscr{R} \rightarrow v(x, y)$ with $\sigma(x, y)=\operatorname{Ad} v(x, y)$. The unitaries $v(x, y)$ may be chosen to take a very special form when $\mathscr{D} \subseteq \mathscr{K}^{\prime}$ is a Cartan subalgebra, but this does not concern us yet; for the moment all we will need is the fact from [13] that $\mathscr{K}=\Gamma_{\mu}^{\infty}(\sigma)$, that is, those essentially bounded operator fields $x \in X \rightarrow T(x) \in B\left(\mathscr{K}_{x}\right)$ satisfying

$$
\sigma(x, y)(T(y))=T(x) \text { a.e. on } \mathscr{R}
$$

(identified up to null sets), acting on $\mathscr{H}$ in the obvious way. (See also [9].) We note also that $(\mathscr{R}, \mu)$ may be assumed to be a discrete measured equivalence 
relation, as in [6]. In addition, the unitaries $\left\{a_{h}: h \in H\right\}$ admit descriptions of the form

$$
\left(a_{h} \xi\right)(x)=\delta(x, h) a(x, h) \xi(x h),
$$

where $(x, h) \in X \times H \rightarrow x h$ is a point realization of the action $h \in H \rightarrow \alpha_{h \mid \mathscr{E}}$ of $H$ on $\mathscr{C}, a(x, h): \mathscr{H}_{x h} \rightarrow \mathscr{X}_{x}$ is unitary, and $\delta(x, h)$ denotes (as it will, throughout) the appropriate Radon-Nikodym derivative.

\section{LEMMA 3.3. We may assume that}

$$
\pi_{x} \circ \alpha_{h}=\operatorname{Ad} a(x, y) \circ \pi_{x h} \quad \text { on } X \times H \text {. }
$$

ProOF. By $[5,8.2 .4]$, the desired relation holds a.e. in $x$ for each $h \in H$; the result follows after deletion of a null set from $X$.

Note that $(x, h) \rightarrow x h$ now provides an action of $H$ on $(\mathscr{R}, \mu)$ as in $\S 2-$ we have $(x, y) \in \mathscr{R} \Leftrightarrow \pi_{x} \stackrel{\sim}{\sim} \pi_{y} \Leftrightarrow \pi_{x} \circ \alpha_{h} \stackrel{u}{\sim} \pi_{y} \circ \alpha_{h} \Leftrightarrow(x h, y h) \in \mathscr{R}$ for all $h \in H$. Further, $N$ acts as inner automorphisms.

We return now to the twisted covariance algebra $(\mathscr{A}, H, \alpha, t)$, introduced above and let $\hat{\pi}_{x}$ denote the induced representation Ind $\pi_{x}$ of the twisted covariance algebra $B=C^{*}(\mathscr{A}, H, \alpha, t)$; recall from [7] that $\hat{\pi}_{x}$ acts on the Hilbert space $L_{N}^{2}\left(H, \mathscr{X}_{x}\right)$ of measurable (classes of) functions $\xi: H \rightarrow \mathscr{H}_{x}$ satisfing $\xi(n h)=\pi_{x}\left(t_{n}\right) \xi(h), n \in N$, and $\int_{H / N}|\xi(h)|^{2} d h<\infty$, and that the action is determined by the operators

$$
\begin{gathered}
\left(\hat{\pi}_{x}(a) \xi\right)(h)=\pi_{x}\left(\alpha_{h}(a)\right) \xi(h), \quad a \in \mathscr{A}, \\
\left(\hat{\pi}_{x}\left(h_{1}\right) \xi\right)(h)=\xi\left(h h_{1}\right), \quad h_{1} \in H .
\end{gathered}
$$

LEMMA 3.4. For $\xi \in L_{N}^{2}\left(H ; \mathscr{H}_{y h}\right)$ and $(x, y, h) \in \mathscr{R} \times_{s} H$, define

$$
\left(V_{N}(x, y, h) \xi\right)(g)=a(x, h) v(x h, y h) \xi\left(h^{-1} g\right) \text {. }
$$

Then

(a) $V_{N}(x, y, h)$ is a unitary operator from $L_{N}^{2}\left(H ; \mathscr{H}_{y h}\right)$ to $L_{N}^{2}\left(H ; \mathscr{H}_{x}\right)$;

(b) $\operatorname{Ad} V_{N}(x, y, h) \circ \hat{\pi}_{y h}=\hat{\pi}_{x}$;

(c) $(x, y, h) \rightarrow V_{N}(x, y, h)$ is a projective representation of $\mathscr{R} \times{ }_{s} H$;

(d) for $n \in N, V_{N}\left(x, x n^{-1}, n\right)$ is a scalar.

ProOF. (a) For $n \in N$ and $\xi \in L_{N}^{2}\left(H ; \mathscr{H}_{y h}\right)$ we have

$$
\begin{aligned}
\left(V_{N}(x, y, h) \xi\right)(n g) & =a(x, h) v(x h, y h) \xi\left(h^{-1} n h h^{-1} g\right) \\
& =a(x, h) v(x h, y h) \pi_{y h}\left(t_{h^{-1} n h}\right) \xi\left(h^{-1} g\right) \\
& =a(x, h) \pi_{x h}\left(t_{h^{-1} n h}\right) v(x h, y h) \xi\left(h^{-1} g\right) \\
& =\pi_{x}\left(\alpha_{h}\left(t_{h^{-1} n h}\right)\right) a(x, h) v(x h, y h) \xi\left(h^{-1} g\right) \\
& =\pi_{x}\left(t_{n}\right)\left(V_{N}(x, y, h) \xi\right)(g) .
\end{aligned}
$$


(b) This follows from routine calculation of $\operatorname{Ad} V_{N}(x, y, h) \circ \hat{\pi}_{y h}(a)$ and $\operatorname{Ad} V_{N}(x, y, h) \circ \hat{\pi}_{y h}(g)$ for $a \in \mathscr{A}$ and $g \in H$.

(c) For $((x, y, g),(y g, z g, h)) \in\left(\mathscr{R} \times_{s} G\right)^{2}$ and $\left.\xi \in L_{N}^{2}\left(H ; \mathscr{H}_{z g h}\right)\right)$

$$
\begin{aligned}
& \left(V_{N}(x, y, g) V_{N}(y g, z g, h) \xi\right)(k) \\
& \quad=a(x, g) v(x g, y g) a(y g, h) v(y g h, z g h) \xi\left(h^{-1} g^{-1} k\right),
\end{aligned}
$$

while $\left(V_{N}(x, z g h) \xi\right)(k)=a(x, g h) v(x g h, z g h) \xi\left(h^{-1} g^{-1} k\right)$. However, both $a(x, g) v(x g, y g) a(y g, h) v(y g h, z g h)$ and $a(x, g h) v(x g h, z g h)$ implement equivalences between the irreducible representations $\pi_{z g h}$ and $\pi_{x} \circ \alpha_{g h}$, so the assertion is verified.

(d) For $n \in N$ and $\xi \in L_{N}^{2}\left(H ; \mathscr{H}_{x}\right)$, we have

$$
\left.\left(V_{N}\left(x, x n^{-1}, n\right) \xi\right) h\right)=a(x, n) v(x n, x) \pi_{x}\left(t_{n}\right)^{*} \xi(h) ;
$$

however

$$
\begin{aligned}
\operatorname{Ad} a & (x, n) v(x n, x) \pi_{x}\left(t_{n}\right)^{*} \circ \pi_{x} \\
& =\operatorname{Ad} a(x, n) v(x n, n) \circ \pi_{x} \circ \alpha_{n}^{-1} \\
& =\operatorname{Ad} a(x, n) \circ \pi_{x n} \circ \alpha_{n}^{-1} \\
& =\pi_{x} \circ \alpha_{n} \circ \alpha_{n}^{-1}=\pi_{x},
\end{aligned}
$$

so $V_{N}\left(x, x n^{-1}, n\right)$ is indeed scalar.

In view of the identity $\left(x, y n^{-1}, n g\right)=\left(x, x n^{-1}, n\right)(x, y, g)$ in $\mathscr{R} \times{ }_{s} H$, and Lemma 3.4(c) and (d), there is a unique representation $\hat{\sigma}$ of the groupoid $\mathscr{R} \vee Q$ of $\S 2$ determined by

$$
\hat{\sigma} \circ p(x, y, h)=\operatorname{Ad} V_{N}(x, y, h)
$$

THEOREM 3.5. The algebras $\Gamma_{\mu}^{\infty}(\hat{\sigma})$ and $\mathscr{M} \rtimes_{\alpha, \omega} Q$ are unitarily equivalent.

ProOF. Note first that there is a unitary $U_{x}: L_{N}^{2}\left(H ; \mathscr{H}_{x}\right) \rightarrow l^{2}\left(Q ; \mathscr{H}_{x}\right)$ given by

$$
\left(U_{x}\right)(s)=\xi(s):\left(U_{x}^{*} \eta\right)(n s)=\pi_{x}\left(t_{n}\right) \eta(s)
$$

for $\xi \in L_{N}^{2}\left(H ; \mathscr{X}_{x}\right), \eta \in l^{2}\left(Q ; \mathscr{H}_{x}\right)$, and we identify $n \in N$ with $(n, e)$ in $H$, and $s \in Q$ with $(1, s) \in H$. We let $U=\int_{X}^{\oplus} U_{x} d \mu(x)$, so $U: L_{N}^{2}(H ; \mathscr{H}) \rightarrow l^{2}(Q ; \mathscr{H})$ is unitary; note that $\operatorname{Ad} U_{x} V_{N}(x, y, e) U_{y}^{*}=v(x, y) \otimes 1$. Thus if $T \sim T(x) \in \Gamma_{\mu}^{\infty}(\hat{\sigma})$ and $S=U T U^{*} \sim U_{x} T(x) U_{x}^{*}$, we have

$$
S \in \Gamma_{\mu}^{\infty}(\sigma \otimes \iota)=\Gamma_{\mu}^{\infty}(\sigma) \otimes B\left(l^{2}(Q)\right),
$$

using [13]. Note also that every element of $\mathscr{R} \times{ }_{s} H$ is a product of elements of the form $(x, y, e),\left(x, x n^{-1}, n\right)$ for $n \in N$, and

$$
\left(x, x,(1, s)^{-1}\right)=\left(x, x,\left(\omega\left(s, s^{-1}\right)^{*}, s^{-1}\right)\right),
$$




$$
\begin{aligned}
\left(U_{x} V_{N}\right. & \left.\left(x, x,\left(1, s^{-1}\right)^{-1}\right) U_{x\left(1, s^{-1}\right)^{-1}}^{*} \xi\right)(t) \\
& \left.=a\left(x,\left(1, s^{-1}\right)^{-1}\right)\left(U_{x\left(1, s^{-1}\right.}^{*}\right)^{-1} \xi\right)\left(\left(1, s^{-1}\right)(1, t)\right) \\
& =a\left(x,\left(1, s^{-1}\right)^{-1}\right)\left(U_{x\left(1, s^{-1}\right)^{-1}}^{*} \xi\right)\left(\omega\left(s^{-1}, t\right), s^{-1} t\right) \\
& =a\left(x,\left(1, s^{-1}\right)^{-1}\right) \pi_{x}\left(\omega\left(s^{-1} t\right)\right) \xi\left(s^{-1} t\right) .
\end{aligned}
$$

Since, in general $a(x, g) a(x g, h)=a(x, g h)$ on $X \times H \times H$, we have

$$
a\left(x,\left(1, s^{-1}\right)^{-1}\right)=a\left(x\left(1, s^{-1}\right)^{-1},\left(1, s^{-1}\right)\right)^{*},
$$

so that if

$$
\left(V_{N}(s) \xi\right)(x)=\delta\left(x,\left(1, s^{-1}\right)^{-1}\right) V_{N}\left(x, x,\left(1, s^{-1}\right)^{-1}\right) \xi\left(x\left(1, s^{-1}\right)^{-1}\right),
$$

we have $U V_{N}(s) U^{*}=\lambda^{\omega}(s)$ as defined in the proof of Lemma 3.2(b), and $U \Gamma_{\mu}^{\infty}(\hat{\sigma}) U^{*}=\mathscr{M} \otimes B\left(l^{2}(Q)\right) \cap\left\{\lambda^{\omega}(s): s \in Q\right\}^{\prime}=\mathscr{M} \rtimes_{\alpha, \omega} Q$, by Lemma 3.2(b).

We now seek to realise $\hat{\sigma}$ as (equivalent, via $\left\{U_{x}\right\}$, to) $\operatorname{Ad} L^{\hat{f}}$ for a suitable $\hat{f} \in Z_{\mu}^{2}(\mathscr{R} \vee Q)$, using the full strength of the assumption that $\mathscr{C}$ is a Cartan subalgebra. For $\mathscr{G}=\mathscr{R}, \mathscr{R} \times{ }_{s} H$ or $\mathscr{R} \vee Q$, and $f \in Z_{\mu}^{2}(\mathscr{G})$ define operators $L^{f}(\gamma): l^{2}\left(r^{-1}(s(\gamma)) \rightarrow l^{2}\left(r^{-1}(r(\gamma))\right.\right.$ and $K^{f}(\gamma): l^{2}\left(s^{-1}(s(\gamma)) \rightarrow l^{2}\left(s^{-1}(r(\gamma))\right.\right.$ by

$$
\begin{aligned}
\left(L^{f}\left(\gamma_{1}\right) \xi\right)\left(\gamma_{2}\right) & =f\left(\gamma_{2}^{-1}, \gamma_{1}\right) \xi\left(\gamma_{1}^{-1} \gamma_{2}\right), \\
\left(K^{f}\left(\gamma_{1}\right) \xi\right)\left(\gamma_{2}\right) & =\delta\left(\gamma_{1}\right)^{\frac{1}{2}} f\left(\gamma_{1},\left(\gamma_{2} \gamma_{1}\right)^{-1}\right)^{-1} \xi\left(\gamma_{2} \gamma_{1}\right)
\end{aligned}
$$

Note that

$$
\begin{gathered}
L^{f}\left(\gamma_{1}\right) L^{f}\left(\gamma_{2}\right)=f\left(\gamma_{1}, \gamma_{2}\right) L^{f}\left(\gamma_{1} \gamma_{2}\right) \quad \text { and } \\
K^{f}\left(\gamma_{1}\right) K^{f}\left(\gamma_{2}\right)=f\left(\gamma_{1}, \gamma_{2}\right)^{-1} K^{f}\left(\gamma_{1} \gamma_{2}\right)
\end{gathered}
$$

on $\mathscr{R}^{(2)}$. Since $\mathscr{C}$ is a Cartan subalgebra, we know from [6] that $\sigma=\operatorname{Ad} v=$ Ad $L^{f}$ for some $f \in Z_{\mu}^{2}(\mathscr{R})$ determined, up to cohomology, by the equation $v(x, y) v(y, z)=f(x, y, z) v(x, z)$ on $\mathscr{R}^{2}$. Furthermore,

$$
(J \xi)(x, y)=\delta(x, y)^{1 / 2} \overline{\xi(y, x)}
$$

is a modular involution for $\mathscr{M}$, and

$$
\mathscr{C}=\left\{S_{\psi}: \psi \in L^{\infty}(X, \mu)\right\}, \quad \mathscr{R}=\left\{R_{\psi}: \psi \in L^{\infty}(X, \mu)\right\},
$$

where $\left(S_{\psi} \xi\right)(x, y)=\psi(y) \xi(x, y)$ and $\left(R_{\psi} \xi\right)(x, y)=\psi(x) \xi(x, y)$. Also, since $J a_{h} J=a_{h}, \operatorname{Ad} a_{h}$ normalises both $\mathscr{C}$ and $\mathscr{D}$, so that

$$
\left(a_{h} \xi\right)(x, y)=\delta(x, h) a_{h}(x, y) \xi(x h, y h)
$$

for some Borel functions $a_{h}: \mathscr{R} \rightarrow \mathbf{T}$. In addition there are Borel maps $t_{n}: X \rightarrow$ T with

$$
\left(t_{n} \xi\right)(x, y)=t_{n}(y) f(y, y n, x)^{-1} \xi(x, y n), \quad n \in N .
$$

To see this, observe that unitaries $w_{n}$ of the indicated form lie in $\Gamma_{\mu}^{\infty}\left(\operatorname{Ad} L^{f}\right)$, and $\operatorname{Ad} w_{n}\left(S_{\phi}\right)=\operatorname{Ad} t_{n}\left(S_{\phi}\right)$ for all $\phi \in L^{\infty}(X, \mu)$; the desired expression for $t_{n}$ follows since $\mathscr{C}$ is maximal abelian in $\Gamma_{\mu}^{\infty}\left(\operatorname{Ad} L^{f}\right)$. 
PROPOSITION 3.6. The functions $a_{g}, t_{n}$ satisfy the relations (a.e.)

(a) $a_{g}(x, y)=a_{g}(y, x)^{-1}$ and $a_{g}(x, x)=1$ for $g \in H$;

(b) $a_{g}(x, y) a_{h}(x g, y h)=a_{g h}(x, y)$ for $g, h \in H$;

(c) $\left(f^{g} \cdot f^{-1}\right)(x, y, z)=\partial a_{g}(x, y, z)$ for $g \in H$;

(d) $a_{n}(x, y)=t_{n}(y) t_{n}(x)^{-1} f(x, x n, y n) f(y, y n, x)^{-1}$ for $n \in N$;

(e) $t_{n}(x h)=t_{n h n^{-1}}(x) a_{h}\left(x, x h n h^{-1}\right)$ for $h \in H, n \in N$.

ProOF. (a) This follows from the identity $J a_{g} J=a_{g}$ and the observation that $a_{g} \xi_{0}=\xi_{0}$, where $\xi_{0}$ is the characteristic function of the diagonal.

(b) This is a consequence of the identity $a_{g} a_{h}=a_{g h}$.

(d) Follows from the identity $a_{n}=t_{n} J t_{n} J$, and the known form of $t_{n}$.

(e) Recall that $\alpha_{g}\left(t_{n}\right)=t_{g n g^{-1}}$; we thus obtain

$a_{g}(x, y) t_{n}(y g) f(y g, y g n, x g)^{-1} a_{g}\left(x, y g n g^{-1}\right)^{-1}=t_{n}(y) f\left(y, y g n g^{-1}, n\right)^{-1}$.

Using (c), (to be proven below), and the skew-symmetry of $a_{g}$, we obtain

$$
t_{n}(y g) a_{g}\left(y, y g n g^{-1}\right)^{-1}=t_{n}(y)
$$

as claimed.

(c) Observe that the unitaries $a(x, g)$ introduced already are given by

$$
(a(x, g) \xi)(y)=a_{g}(x, y) \xi(y g) .
$$

Note that if $b \in \mathscr{A} \subseteq \Gamma_{\mu}^{\infty}\left(\operatorname{Ad} L^{f}\right)$, then $c=a_{g} b a_{g}^{*}$ is in $\Gamma_{\mu}^{\infty}\left(\operatorname{Ad} L^{f}\right)$, so that

$$
\begin{aligned}
\operatorname{Ad} L^{f}(x, y) a(y, g) \circ \pi_{y g}(b) & =\operatorname{Ad} L^{f}(x, y) \pi_{y}(c)=\pi_{x}(c) \\
& =\operatorname{Ad} a(x, g) L^{f}(x g, y g) \circ \pi_{y g}(b) .
\end{aligned}
$$

Since $\pi_{y g}$ is irreducible, we obtain

$$
L^{f}(x, y) a(y, g)=d_{g}(x, y) a(x, g) L^{f}(x g, y g)
$$

for some Borel map $d_{g}: \mathscr{R} \rightarrow \mathbf{T}$. From this we see

$$
f(z, x, y) a_{g}(y, z)=d_{g}(x, y) a_{g}(x, z) f(z g, y g, x g)
$$

a.e. on $\mathscr{R}^{(2)} ;$ taking $z=x$ and noting $a_{g}(x, x)=1=f(x, x, y)=f(x g, x g, y g)$, we obtain $d_{g}(x, y)=a_{g}(y, x)$. Thus we have

$$
\begin{aligned}
\left(f^{g} \cdot f^{-1}\right)(z, x, y) & =a_{g}(y, z) a_{g}(y, x)^{-1} a_{g}(x, z)^{-1} \\
& =a_{g}(z, x) a_{g}(x, y) a_{g}(z, y)^{-1}
\end{aligned}
$$

Proposition 3.6(a), (b), (c) and Theorem 2.2 together guarantee that the formula

$$
F((x, y, g),(y g, z g, h))=f(x g h, y g h, z g h) a_{h}^{-1}(x g, y g)
$$

defines an element of $Z_{\mu}^{2}\left(\mathscr{R} \times_{s} H\right)$. 
LEMMA 3.7. (a) $\left(V_{N}(x, y, g) \xi\right)(z, h)=F\left((x, z, h)^{-1},(x, y, g)\right) \xi\left(z g, g^{-1} h\right)$ on $L_{N}^{2}\left(H ; \mathscr{H}_{y g}\right)$;

(b) $V_{N}\left(x, x n^{-1}, n\right)=t_{n}(x)^{-1}, n \in N$.

ProOF. (a) With the notation as already established

$$
\begin{aligned}
\left(V_{N}(x, y, g) \xi\right)(z, h) & =\left(a(x, g) L^{f}(x g, y g) \xi\right)\left(z, g^{-1} h\right) \\
& =a_{g}(x, z)\left(L^{f}(x g, y g) \xi\right)\left(z g, g^{-1} h\right) \\
& =a_{g}(x, z) f(z g, x g, y g) \xi\left(z g, g^{-1} h\right)
\end{aligned}
$$

while

$$
F\left((x, z, h)^{-1},(x, y, g)\right)=F\left(\left(z h, x h, h^{-1}\right),(x, y, g)\right)=f(z g, x g, y g) a_{g}(z, x)^{-1} .
$$

The conclusion follows, using Proposition 3.6(a).

$$
\left(V_{N}\left(x, x n^{-1}, n\right) \xi\right)(h)=a(x, n) L^{f}(x n, x) \pi_{x}\left(t_{n}\right)^{*} \xi(h)
$$

as in Lemma 3.4(d). However for $\eta \in \mathscr{H}_{x}=l^{2}\left(r^{-1}(x)\right)$, $\left(a(x, n) L^{f}(x n, x) \pi_{x}\left(t_{n^{-1}}\right) \eta\right)(y)=a_{n}(x, y) f(y n, x n, x) t_{n^{-1}}(y n) f(y n, y, x)^{-1} \eta(y)$. But $t_{n} t_{n^{-1}}=1$, so that $t_{n^{-1}}(y n)=t_{n}(y)^{-1}$; using also the form given for $a_{n}$ in Proposition 3.6(d), and the cocycle identity for $f$ with the variables $y n, y, x n$ and $x$, we conclude

$$
a(x, y) L^{f}(x n, x) \pi_{x}\left(t_{n}\right)^{*}=t_{n}(x)^{-1} .
$$

We thus see, that in the case when $N$ is trivial (so that we had started with an action of $Q$ on $\mathscr{M}$ ) that $\hat{\sigma}=\operatorname{Ad} L^{F}$. Our final conclusion is that $F$ defines a cocycle $\hat{f}$ on $\mathscr{R} \vee Q$, and $\hat{\sigma}$ is (equivalent to) $\operatorname{Ad} L^{\hat{f}}$. For the purpose of this proof we use the notation $h_{s}=(1, s) \in H$.

THEOREM 3.8. With the notation already established, the formula

$$
\begin{aligned}
& \hat{f}\left((x, y, s),\left(y h_{s}, z h_{s}, t\right)\right) \\
&= f\left(x h_{s^{-1}}^{-1} h_{t^{-1}}^{-1}, y h_{s} h_{t^{-1}}^{-1}, z h_{s} h_{t}\right) a_{h_{s^{-1}}^{-1}}\left(x h_{s^{-1}}^{-1}, y h_{s}\right)^{-1} \\
& \times f\left(x h_{s^{-1}}^{-1} h_{t^{-1}}^{-1}, x h_{t^{-1} s^{-1}}^{-1}, z h_{s} h_{t}\right)^{-1} \\
& \times a_{h_{t^{-1},-1}^{-1}}\left(x h_{s^{-1}}^{-1} h_{t^{-1}}^{-1} h_{t^{-1} s^{-1}}, x\right) t_{h_{s^{-1}}^{-1} h_{t^{-1}}^{-1} h_{t^{-1}}-1}(x)^{-1}
\end{aligned}
$$

defines an element of $Z_{\mu}^{2}(\mathscr{R} \vee Q)$; further, $\hat{\sigma}$ is equivalent, via the unitaries $U_{x}$ of Theorem 3.5, with $\operatorname{Ad} L^{\hat{f}}$, as automorphic representations of $\mathscr{R} \vee Q$.

ProOF. Note the map $(x, y, s) \in \mathscr{R} \vee Q \rightarrow\left(x, y h_{s} h_{s^{-1}}, h_{s^{-1}}^{-1}\right) \in \mathscr{R} \times_{s} H$ provides a section for the quotient map $\mathscr{R} \times{ }_{s} H \rightarrow \mathscr{R} \vee Q$. Since $V_{N}$ is a 
projective representation with multiplier $F$ (Lemma 3.7(a)), we have

$$
\begin{gathered}
V_{N}\left(x, y h_{s} h_{s^{-1}}, h_{s^{-1}}^{-1}\right) V_{N}\left(y h_{s}, z h_{s} h_{t} h_{t^{-1}}, h_{t^{-1}}^{-1}\right) \\
=F\left(\left(x, y h_{s} h_{s^{-1}}, h_{s^{-1}}^{-1}\right),\left(y h_{s} z h_{s} h_{t} h_{t^{-1}}, h_{t^{-1}}^{-1}\right) .\right. \\
V_{N}\left(x, z h_{s} h_{t} h_{t^{-1}} h_{s^{-1}}, h_{s^{-1}}^{-1} h_{t^{-1}}^{-1}\right) \\
=F\left(\left(x, y h_{s} h_{s^{-1}}, h_{s^{-1}}^{-1}\right),\left(y h_{s}, z h_{s} h_{s^{-1}}, h_{s^{-1}}^{-1}\right)\right) \\
\times F\left(\left(x, x h_{t^{-1} s^{-1}}^{-1} h_{t^{-1}} h_{s^{-1}}, h_{s^{-1}}^{-1}, h_{t^{-1}}^{-1} h_{t^{-1}} s^{-1}\right) t_{h_{s^{-1}} h_{t^{-1}}^{-1} h_{t^{-1}}-1}(x)^{-1}\right. \\
\times V_{N}\left(x, z h_{s} h_{t} h_{t^{-1} s^{-1}}, h_{t^{-1}}^{-1} s^{-1}\right)
\end{gathered}
$$

where we have used the identity

$$
\begin{aligned}
& \left(x, z h_{s} h_{t} h_{t^{-1}} h_{s^{-1}}, h_{s^{-1}}^{-1} h_{t^{-1}}^{-1}\right) \\
& \quad=\left(x, x h_{t^{-1} s^{-1}}^{-1} h_{t^{-1}} h_{s^{-1}}, h_{s^{-1}}^{-1} h_{t^{-1}}^{-1} h_{t^{-1} s^{-1}}\right),\left(x, z h_{s} h_{t} h_{t^{-1} s^{-1}}, h_{t^{-1} s^{-1}}^{-1}\right)
\end{aligned}
$$

and Lemma 3.7(b), with $n=h_{s^{-1}}^{-1} h_{t^{-1}}^{-1} h_{t^{-1}} s^{-1}$. However, the above coefficient of $V_{N}\left(x, x h_{s} h_{t} h_{t^{-1} s^{-1}}, h_{t^{-1} s^{-1}}^{-1}\right)$ is precisely $\hat{f}\left((x, y, s),\left(y h_{s}, z h_{s}, t\right)\right)$ as defined is the statement of the theorem, so that $\hat{f} \in Z_{\mu}^{2}(\mathscr{R} \vee Q)$-the masochistic reader may also check this by direct calculation.

Note that by definition we have

$$
\begin{gathered}
\hat{f}\left((x, z, t)^{-1},(x, y, s)=f\left(z h_{s^{-1}}^{-1}, x h_{x^{-1}}^{-1}, y h_{s}\right) f\left(z h_{s^{-1}}^{-1}, z h_{t} h_{s^{-1}}^{-1}, y h_{s}\right)^{-1}\right. \\
\times a_{h_{o-1}^{-1}}(z, x)^{-1} a_{h_{t^{-1}}^{-1}}\left(z h_{s^{-1}}^{-1} h_{s^{-1} t}, z h_{t}\right) t_{h_{t}^{-1} h_{g^{-1}}^{-1} h_{s^{-1}}}\left(z h_{t}\right)^{-1} .
\end{gathered}
$$

If $U_{x}$ is as in the proof of Theorem 3.5,

$$
\begin{aligned}
& \left(U_{x} V_{N}\left(x, y h_{s} h_{s^{-1}}, h_{s^{-1}}^{-1}\right) U_{y h_{o}}^{*} \xi\right)(t) \\
& \quad=a\left(x, h_{s^{-1}}^{-1}\right) L^{f}\left(x h_{s^{-1}}^{-1}, y h_{s}\right)\left(U_{y h_{o}}^{*} \xi\right)\left(h_{s^{-1}} h_{t} h_{s^{-1}}^{-1} h_{s^{-1}}\right) \\
& \quad=a\left(x, h_{s^{-1}}^{-1}\right) L^{f}\left(x h_{s^{-1}}^{-1}, y h_{s}\right) \pi_{y h_{o}}\left(\omega\left(s^{-1}, t\right)\right) \xi\left(s^{-1} t\right)
\end{aligned}
$$

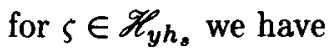

$$
\begin{aligned}
\left(a\left(x, h_{s^{-1}}^{-1}\right) L^{f}\left(x h_{s^{-1}}^{-1}, y h_{s}\right) \pi_{y h_{s}}\left(\omega\left(s^{-1}, t\right)\right) \zeta\right)(z) \\
=a_{h_{s^{-1}}}(x, z) f\left(z h_{s^{-1}}^{-1}, y h_{s}\right) t_{\omega\left(s^{-1}, t\right)}\left(z h_{s^{-1}}^{-1}\right) \\
\quad \times f\left(z h_{s^{-1}}^{-1}, z h_{t} h_{s^{-1}}^{-1}, y h_{s}\right)^{-1} \zeta\left(z h_{t} h_{s^{-1} t}^{-1}\right) .
\end{aligned}
$$

However, using the fact that $t_{h_{t}^{-1} h_{g^{-1}}^{-1} h_{s-1}}\left(z h_{t}\right)^{-1}=t_{h_{-1}^{-1} h_{s-1} h_{t}}\left(z h_{s^{-1}}^{-1} h_{s^{-1}}^{-1}\right)$, and Proposition 3.6(e) with $x=z h_{s^{-1}}^{-1} h_{s^{-1}} t, g=h_{s^{-1} t}^{-1}$ and $n=h_{s^{-1}} h_{t} h_{s^{-1}}^{-1}$, this last expression reduces to $\hat{f}((x, z, t),(x, y, s))_{\varsigma}\left(z h_{t} h_{s^{-1} t}^{-1}\right)$.

We thus have the desirable, but not unexpected, result that $\mathscr{M} \rtimes_{\alpha, \omega} Q=$ $\Gamma_{\mu}^{\infty}\left(\operatorname{Ad} L^{\hat{f}}\right)$ for some specifically determined $\hat{f} \in Z_{\mu}^{2}(\mathscr{R} \vee Q)$. The author believes that this fact should have a much more simple proof than the one given here; 
despite many attempts, even in the presence of some simplifying assumptions, a simple, "conceptual" proof has not been found.

Another important feature of the analysis so far is that the generators $\hat{\pi}(a)$, $\hat{\pi}(s)$, for $a \in \mathscr{A}$ and $s \in Q$, of the representation Ind $\iota$ of $\mathscr{B}=C^{*}(\mathscr{A}, H, \alpha, t)$ admit explicit decomposition over $\mathscr{D} \otimes 1$ of the form

$$
\begin{aligned}
& \left(\hat{\pi}_{x}(a) \xi\right)(t)=\pi_{x}\left(\alpha_{t}(a)\right) \xi(t), \quad a \in \mathscr{A}, \\
& \left(\hat{\pi}_{x}(s) \xi\right)(t)=\pi_{x}(\omega(t, s)) \xi(t s .), \quad s \in Q .
\end{aligned}
$$

Here, we have realized the representations $\hat{\pi}_{x}$ of the discussion preceding Lemma 3.4 on $l^{2}\left(Q ; \mathscr{H}_{x}\right)$ via the unitary $U_{x}$. We shall further analyze these representations in the next section.

\section{Analysis of the representations $\hat{\pi}_{x}$}

In general the representations $\hat{\pi}_{x}$ are not irreducible, we seek here information as to the precise extent to which this irreducibility fails.

We consider, as in $\S 3$, the twisted covariance system $(\mathscr{A}, H, \alpha, t)$; we define $H_{x}=\left\{h \in H: \pi_{x} \circ \alpha_{h} \sim \pi_{x}\right\}$, and $Q_{x}=H_{x} / N \subseteq Q$. Let $\phi_{x}=\operatorname{Ind}_{N}^{H_{x}} \pi_{x}$ be the induced representation of the twisted covariance algebra $\mathscr{B}_{x}=C^{*}\left(\mathscr{A}, H_{x}, \alpha, t\right)$, acting on $L_{N}^{2}\left(H_{2}, \mathscr{H}_{x}\right)$. Unfortunately, Green's notion of induced representation does not allow us to consider $\operatorname{Ind}_{H_{x}}^{H} \phi_{x}$, since $H_{x}$ may fail to be normal in $H$. Even so, one may consider the Hilbert space $L_{H_{x}}^{2}\left(H ; L_{N}^{2}\left(G_{x}, \mathscr{H}_{x}\right)\right)$ as a space of appropriately invariant $\mathscr{H}_{x}$-valued functions on $H \times G_{x}$, where the action on $H$ is via the induced representation, and this Hilbert space is unitarily equivalent to $L_{N}^{2}\left(H ; \mathscr{H}_{x}\right)$ via the unitary $P_{x}$ defined (formally) by

$$
\left(P_{x} \xi\right)(h)=\xi(h, e), \quad\left(P_{x}^{*} \eta\right)\left(h_{1}, h_{2}\right)=\eta\left(h_{2} h_{1}\right)
$$

for $\xi \in L_{H_{x}}^{2}\left(H ; L_{N}^{2}\left(H_{x} ; \mathscr{H}_{x}\right)\right)$ and $\eta \in L_{N}^{2}\left(H ; \mathscr{H}_{x}\right)$. Also, if $T \in \phi_{x}\left(B_{x}\right)^{\prime}$ on $L_{N}^{2}\left(H_{x} ; \mathscr{H}_{x}\right)$, we define

$$
\kappa_{x}(T)=P_{x} \tilde{T} P_{x}^{*}
$$

where $\tilde{T}$ acts on $L_{H_{x}}^{2}\left(H ; L_{N}^{2}\left(H_{x}, \mathscr{H}_{x}\right)\right)$ via $(\tilde{T} \xi)(h)=T \xi(h)$ for $h \in H$. It is routine to check that $\kappa_{x}(T) \in \hat{\pi}_{x}(B)^{\prime}$ on $L_{N}^{2}\left(H ; \mathscr{H}_{x}\right)$.

THEOREM 4.1. For almost all $x, \kappa_{x}$ is an isomorphism.

The proof is given in the sequence of lemmas which follow.

For $s \in Q_{x}$, we choose a unitary $v_{s}$ on $\mathscr{H}_{x}$ with

$$
\operatorname{Ad} v_{s} \circ \pi_{x}=\pi_{x} \circ \alpha_{s}
$$

note $v_{s} v_{t}=c_{x}(s, t) \pi_{x}(\omega(s, t)) v_{s t}$ on $Q_{x} \times Q_{x}$, where $c_{x}(s, t) \in \mathbf{T}$. Clearly $c_{x} \in$ $Z^{2}\left(H_{x} ; \mathbf{T}\right)$. 
LEMMA 4.2. Define $V_{x}$ on $l^{2}\left(Q_{x} ; \mathscr{H}_{x}\right)$ by $\left(V_{x} \xi\right)(s)=v_{s}^{*} \xi(s)$. Then Ad $v_{x}$ 。 $\phi_{x}\left(\mathscr{B}_{x}\right)^{\prime}$ is generated by $\left\{1 \otimes \lambda^{c_{x}}(s): s \in Q_{x}\right\}$, where $\lambda^{c_{x}}$ is the left regular $c_{x}$ representation of $Q_{x}$, defined by

$$
\left(\lambda^{c_{x}}(s) \eta\right)(t)=c_{x}\left(s^{-1}, t\right)^{-1} \eta\left(s^{-1} t\right), \quad \eta \in l^{2}\left(Q_{x}\right) .
$$

ProOF. With $\phi_{x}$ realized on $l^{2}\left(Q_{x} ; \mathscr{H}_{x}\right)$ we have, for $a \in \mathscr{A}$

$$
\left(V_{x} \phi_{x}(a) V_{x}^{*} \xi\right)(s)=\operatorname{Ad} v_{s}^{*} \circ \pi_{x} \circ \alpha_{s}(a) \xi(s)=\pi_{x}(a) \xi(s),
$$

and

$$
\begin{aligned}
\left(V_{x} \phi_{x}(t) V_{x}^{*} \xi\right)(s) & =v_{s}^{*} \pi_{x}(\omega(s, t)) v_{s t} \xi(s t) \\
& =c_{x}(s, t)^{-1} v_{t} \xi(s t) \\
& =v_{t}\left(\rho^{\bar{c}_{x}}(t) \xi\right)(s)
\end{aligned}
$$

Thus $\operatorname{Ad} V_{x} \circ \phi_{x}\left(\mathscr{B}_{x}\right)$ is generated on $\mathscr{H}_{x} \otimes l^{2}\left(Q_{x}\right)$ by $\left\{\pi_{x}(a) \otimes 1: a \in \mathscr{A}\right\}$, and $\left\{v_{t} \otimes \rho^{\bar{c}_{x}}(t): t \in Q\right\}$, and the assertion follows.

LEMMA 4.3. $\hat{\pi}_{x}(\mathscr{B})^{\prime}$ is generated on $L_{N}^{2}\left(H ; \mathscr{L}_{x}\right)$ by $\left\{V_{N}\left(x, x h_{s^{-1}}, h_{s^{-1}}^{-1}\right)\right\}$, where $h_{s}$ is as in Theorem 3.8 .

PROOF. Transform $L_{N}^{2}\left(H ; \mathscr{H}_{x}\right)$ to $l^{2}\left(Q_{x} ; \mathscr{H}_{x}\right)$ as usual, so

$$
\begin{aligned}
& \left(\hat{\pi}_{x}(a) \xi\right)(t)=\pi_{x}\left(\alpha_{t}(a)\right) \xi(t), \quad a \in \mathscr{A}, \\
& \left(\hat{\pi}_{x}(s) \xi\right)(t)=\pi_{x}(\omega(t, s)) \xi(t s), \quad t \in Q,
\end{aligned}
$$

and $\left(V_{N}\left(x, x h_{s^{-1}}, h_{s^{-1}}^{-1}\right) \xi\right)(t)=d_{x}(s, t) \xi\left(s^{-1} t\right)$, where

$$
d_{x}(s, t)=a\left(x, h_{s^{-1}}^{-1}\right) L^{f}\left(x h_{g^{-1}}^{-1}, x\right) \pi_{x}\left(\omega\left(s^{-1}, t\right)\right)
$$

is a unitary which conjugates $\pi_{x} \circ \alpha_{s^{-1} t}$ to $\pi_{x} \circ \alpha_{t}$.

Now if $T \in B\left(l^{2}\left(Q ; \mathscr{H}_{x}\right)\right)$ commutes with the above generators, write $T=$ $\left(T_{t, s}\right)$ as a $B\left(\mathscr{H}_{x}\right)$-valued matrix, and we see that

(a) $T_{t, s} \pi_{x}\left(\alpha_{s}(a)\right)=\pi_{x}\left(\alpha_{t}(a)\right) T_{t, s}, a \in \mathscr{A}$, from which it follows, by irreducibility of $\pi_{x}$, that

$$
T_{t, s^{-1} t}=\psi(s, t) d_{x}(s, t)
$$

for some $\psi(s, t) \in \mathbf{T}$. In addition, for $r \in Q_{x}$,

(b) $T_{t r, s r}=\pi_{x}(\omega(t, r))^{*} T_{t, s} \pi_{x}(\omega(s, r))$.

To prove our assertion, it is enough to show that $\psi(s, t)$ is independent of $t$. But

$$
\begin{aligned}
\psi(s, t) & =T_{t, s^{-1} t} d_{x}(s, t)^{*} \\
& =T_{t, s^{-1} t} \pi_{x}\left(\omega\left(s^{-1}, t\right)\right)^{*} L^{f}\left(x h_{s^{-1}}^{-1}, x\right)^{*} a\left(x, h_{s^{-1}}^{-1}\right)^{*},
\end{aligned}
$$


so that, to show $\psi(s, t)=\psi(s, t r)$ it is enough to show

(c) $T_{t, s^{-1} t} \pi_{x}\left(\omega\left(s^{-1}, t\right)\right)^{*}=T_{t r, s^{-1} t r} \pi_{x}\left(\omega\left(s^{-1}, t r\right)\right)^{*}$.

Using (b) above, the right hand side of (c) becomes

$$
\pi_{x}(\omega(t, r))^{*} T_{t, s^{-1} t} \pi_{x}\left(\omega\left(s^{-1} t, r\right)\right) \pi_{x}\left(\omega\left(s^{-1}, t r\right)\right)^{*} .
$$

Applying (a) and the identity $\alpha_{s^{-1}}(\omega(t, r)) \omega\left(s^{-1}, t r\right)=\omega\left(s^{-1}, t\right) \omega\left(s^{-1} t, r\right)$, the desired identity (c) is established.

COMPLETION OF PROOF OF THEOREM 4.1. It suffices to show that

$$
\kappa_{x}\left(V_{x}^{*} \lambda^{c_{x}}(s) V_{x}\right)=V_{N}\left(x, x h_{s^{-1}}, h_{s^{-1}}^{-1}\right) .
$$

With the unitary $V_{x}$ as in the proof of Lemma 4.3 , and $U_{x}: L_{N}^{2}\left(H_{x} ; \mathscr{H}_{x}\right) \rightarrow$ $l^{2}\left(Q_{x} ; \mathscr{H}_{x}\right)$, we have $U_{x}^{*} V_{x}^{*}\left(1 \otimes \lambda^{c_{x}}(s)\right) V_{x} U_{x}=\lambda_{1}(s)$, where

$$
\left(\lambda_{1}(s) \xi\right)(n t)=\pi_{x}\left(t_{n}\right) v_{s^{-1}}^{*} \xi\left(s^{-1} t\right)
$$

The associated operator $\tilde{\lambda}(s)$ on $L_{H_{x}}^{2}\left(H ; L_{N}^{2}\left(H_{x} ; \mathscr{H}_{x}\right)\right)$ is given by $\left(\tilde{\lambda}_{1}(s) \xi\right)(h)$ $=\lambda_{1}(s) \xi(h)$, and if $P_{x}$ is the unitary identifying $L_{H_{x}}^{2}\left(H ; L_{N}^{2}\left(H_{n} ; \mathscr{H}_{x}\right)\right)$ with $L_{N}^{2}\left(H ; \mathscr{X}_{x}\right)$ given in the discussion at the start of $\S 4$, then

$$
\begin{aligned}
\left(P_{x} \tilde{\lambda}_{1}(s) P_{x}^{*} \xi\right)(h) & =v_{s^{-1}}^{*}\left(P_{x}^{*} \xi\right)\left(h, s^{-1}\right)=v_{s^{-1}}^{*} \xi\left(s^{-1} h\right) \\
& =a\left(s, h_{s^{-1}}^{-1}\right) L^{f}\left(x h_{s^{-1}}^{-1}, x\right) \xi\left(h_{s^{-1}} h\right) \\
& =\left(V_{N}\left(x, x h_{s^{-1}}, h_{s^{-1}}^{-1}\right) \xi\right)(h),
\end{aligned}
$$

since we may choose $v_{s}=L^{f}\left(x h_{s}^{-1}, x\right)^{*} a\left(x, h_{s}^{-1}\right)^{*}$.

REMARK 4.4. The cocycle $c_{x}$ used throughout the proof of Theorem 4.1 is nothing but the "Mackey obstruction" associated to the representation $\pi_{x}$ of $\mathscr{A}$, the action $\alpha$ of $H$ on $\mathscr{A}$ and the "little group" $Q_{x}$. Note that by the proof of Theorem 4.1, and the fact (cf. Theorem 3.8) that

$$
L^{f}(x, y, s)=U_{x} V_{N}\left(x, y h_{s} h_{s^{-1}}, h_{s^{-1}}^{-1}\right) U_{y h_{\theta}}^{*},
$$

that on $Q_{x} \times Q_{x}, c_{x}(s, t)=\hat{f}\left(\left(x, x h_{s}^{-1}, s\right),\left(x, x h_{t}^{-1}, t\right)\right)$; thus all the Mackey obstructions appear as restrictions to isotropy groups of a single cocycle $\hat{f} \in$ $Z_{\mu}^{2}(\mathscr{R} \vee Q)$

REMARK 4.5. To obtain a decomposition of the representation $\hat{\pi}=\operatorname{Ind}_{N}^{H} \pi$ of $\mathscr{B}$ into irreducibles, it is evidently sufficient to choose a maximal abelian subalgebra $\mathscr{D}_{x}$ of $1 \otimes \lambda^{c_{x}}\left(Q_{x}\right)^{\prime \prime}$ (or more accurately, of $\left\{V_{N}\left(x, x h_{s}^{-1}, s\right): s \in Q_{x}\right\}^{\prime}$ ) and to decompose the representations $\hat{\pi}_{x}$ over $\mathscr{D}_{x}$. If this can be done in such a way that

(a) $\mathscr{D}_{x}$ is a Cartan subalgebra; 
(b) $\operatorname{Ad} V_{N}(x, y, h)\left(\mathscr{D}_{y h}\right)=\mathscr{D}_{x}$ on $\mathscr{R} \times{ }_{s} H$,

the algebra $\hat{\mathscr{D}}=\int_{X}^{\oplus} \mathscr{D}_{x} d \mu(x)$ is a Cartan subalgebra of $\hat{\pi}(B)^{\prime}$, and the associated decomposition is of the desired kind. Regrettably, we know of no method by which such a "coherent" family of Cartan subalgebras may be found, nor abstract conditions under which such a family exists, although the existence, individually, of Cartan subalgebras in $\lambda^{c_{x}}\left(Q_{x}\right)^{\prime \prime}$ is assured if $Q$ is amenable. However, in the case where $\lambda^{c_{x}}\left(Q_{x}\right)^{\prime \prime}$ is type I, we may use simply the central decomposition and multiplicity theory to effect the desired decomposition.

\section{Applications to group extensions}

We consider an exact sequence $1 \rightarrow K \rightarrow G \rightarrow Q \rightarrow 1$ of locally compact second countable groups, with $Q$ discrete. Let $\mathscr{K}=\rho^{K}(K)^{\prime \prime}$, where $\rho^{K}$ is the right regular representation of $K$ on $L^{2}(K)$. Since $G$ acts on $K$, it acts on $\mathscr{M}$ via an action $\gamma$ where $\gamma_{g}\left(\rho^{K}(k)\right)=\rho^{K}\left(g k g^{-1}\right)$. If $s \in Q \rightarrow g_{s} \in G$ is a section for the surjection $G \rightarrow Q$, and $\nu(s, t)=\rho^{K}\left(g_{s} g_{t} g_{s t}^{-1}\right)$, then we have $\gamma_{s} \circ \gamma_{t}=\operatorname{Ad} \nu(s, t) \gamma_{s t}$, and $\gamma_{r}(\nu(s, t) \nu(r, s t)=\nu(r, s) \nu(r s, t)$, as at the beginning of $\S 2$. We assume that $\left\{\mathscr{K}, \gamma_{s}, \nu\right\}$ satisfies Assumption 3.1 of $\S 3$; note that by the results of $[17, \S 5]$ this hypothesis is satisfied if $G$ is discrete and amenable (in which case the group $H$ of $\S 3$ may be viewed as a subgroup of $G$ ), or if the sequence $1 \rightarrow K \rightarrow G \rightarrow Q \rightarrow 1$ is split and $\mathscr{K}$ is semifinite and injective, and $Q$ is amenable.

In order to apply the results of $\S 3$ and $\S 4$ we need

LEMMA 5.1. (a) The von Neumann algebra $\mathscr{K}(G)$ generated by the right regular representation $\rho^{G}$ of $G$ is unitarily equivalent with $\mathscr{M} \rtimes_{\gamma, \nu} Q$ under the map taking $\rho^{G}(k)$ to $\pi^{\gamma}\left(\rho^{K}(k)\right)$ for $k \in K$, and $\rho^{G}\left(g_{s}\right)$ to $\rho^{\nu}(s)$ for $s \in Q$;

(b) The map $\pi^{\gamma}(x) \rightarrow \pi^{\alpha}(x)$, for $x \in \mathscr{K}$ and $\rho^{\nu}(s) \rightarrow \pi^{\beta}\left(u_{s}^{*}\right) \rho^{\omega}(s)$ for $s \in Q$ extends to a unitary equivalence of $\mathscr{M} \rtimes_{\gamma, \nu} Q$ with $\mathscr{M} \rtimes_{\alpha, \omega} Q$.

Proof. (a) If we identify $G$ with $K \times Q$ via $k g_{s} \rightarrow(k, s)$, the associated unitary $(U \xi)(k, s)=\xi\left(k g_{s}\right)$ from $L^{2}(G)$ to $l^{2}\left(Q ; L^{2}(K)\right)$ conjugates $\rho^{G}(k)$ to $\pi^{\gamma}\left(\rho^{K}(k)\right)$ and $\rho^{G}\left(g_{s}\right)$ to $\rho^{\nu}(s)$ for $k \in K$ and $s \in Q$.

(b) The map $U: l^{2}\left(Q ; L^{2}(K)\right) \rightarrow l^{2}\left(Q ; L^{2}(K)\right)$ given by $(U \xi)(s)=u_{s} \xi(s)$ implements the indicated equivalence.

Now, by Theorem $3.8, \rho^{G}$ is (unitarily equivalent with) a representation which generates $\Gamma_{\mu}^{\infty}\left(\operatorname{Ad} L^{\hat{f}}\right)$; note if $C^{*}(G)$ denotes the grcup $C^{*}$-algebra of 
$G$, both $\rho^{G}\left(C^{*}(G)\right)$ and $\hat{\pi}(B)$ may be viewed as separable, $\sigma$-weakly dense $C^{*}$ subalgebras of $\Gamma_{\mu}^{\infty}\left(L^{\hat{f}}\right)$, so that if

$$
\rho^{G}=\int_{X}^{\oplus} \rho_{x}^{G} d \mu(x)
$$

is the decomposition of $\rho^{G}$ over $\mathscr{D} \otimes 1$, then using the arguments of [18], we may delete a $\mu$-null set from $X$ and assume that $\rho_{x}^{G} \stackrel{u}{\sim} \rho_{y}^{G} \Leftrightarrow \hat{\pi}_{x} \stackrel{u}{\sim} \hat{\pi}_{y}$, and that the intertwining operators between these pairs of representations are precisely the same-indeed we may choose the auxiliary $C^{*}$-algebra $\mathscr{A}$ of $\S 3$ so that $\mathscr{A} \supseteq \rho^{K}\left(C^{*}(K)\right)$.

We now wish to interpret the results of $\S 3, \S 4$ in terms of $K, G$, and the Mackey theory; some care must be taken in this since our results in $\S 3, \S 4$ relate to the auxiliary twisted covariant system $(\mathscr{A}, H, \alpha, t)$, whereas we need interpretation in terms of the system $\left(C^{*}(K), G, \gamma, \tau\right)$, where the normal subgroup for $\tau$ is $K$, and $\tau(k)=\rho^{K}(k)$.

We set $G_{x}=\left\{g \in G: \rho_{x} \circ \gamma_{g} \stackrel{u}{\sim} \rho_{x}\right.$, and note that $G_{x} / K \cong Q_{x}$ since $\rho_{x} \sim \rho_{y} \Leftrightarrow \pi_{x} \sim \pi_{y}$; let $\psi_{x}=\operatorname{Ind}_{K}^{G_{x}} \rho_{x}$ be the (Mackey) induced representation on $L_{K}^{2}\left(G_{x} ; \mathscr{X}_{x}\right)$, and $\hat{\rho}_{x}=\operatorname{Ind}_{K}^{G} \rho_{x}$, on $L_{K}^{2}\left(G ; \mathscr{H}_{x}\right)$

PROPOSITION 5.2. The natural map from $\psi_{x}\left(G_{x}\right)^{\prime}$ to $\hat{\rho}_{x}(G)^{\prime}$ is an isoomorphism for almost all $x$.

PROOF. We have unitary equivalences

$$
L_{K}^{2}\left(G_{x}, \mathscr{H}_{x}\right) \stackrel{u}{\sim} l^{2}\left(Q_{x} ; \mathscr{H}_{x}\right) \stackrel{u}{\sim} L_{N}^{2}\left(H_{x} ; \mathscr{H}_{x}\right)
$$

and

$$
L_{K}^{2}\left(G ; \mathscr{K}_{x}\right) \stackrel{u}{\sim} l^{2}\left(Q ; \mathscr{K}_{x}\right) \stackrel{u}{\sim} L_{H}^{2}\left(N ; \mathscr{K}_{x}\right)
$$

of the form already used; the map in question is just the composition of the corresponding spatial isomorphisms with the map $\kappa_{x}$ of Theorem 4.1, and the result follows.

THEOREM 5.3. Let $1 \rightarrow K \rightarrow G \rightarrow Q \rightarrow 1$ be an exact sequence of locally compact second countable groups with $Q$ discrete, and either $G$ discrete amenable, or $Q$ amenable, $G$ a split extension, and $\rho^{K}(K)^{\prime \prime}$ semifinite injective. Then there is a decomposition

$$
\rho^{K}=\int_{X}^{\oplus} \rho_{x} d \mu(x)
$$

of the right regular representation $\rho^{K}$ of $K$ such that

(i) the diagonal algebra for the decomposition is a Cartan subalgebra of $\rho^{K}(K)^{\prime}$; 
(ii) if $G_{x}=\left\{g \in G: \pi_{x} \circ \gamma_{g} \stackrel{\sim}{\sim} \pi_{x}\right\}, \psi_{x}=\operatorname{Ind}_{K}^{G_{x}} \rho_{x}$ and $\psi_{x}=\int_{U_{x}}^{\oplus} \psi_{x, u} d \mu_{x}(u)$ is a decomposition of $\psi_{x}$ into irreducibles, then

$$
\operatorname{Ind}_{K}^{G} \pi_{x}=\int_{U_{x}}^{\oplus} \operatorname{Ind}_{G_{x}}^{G} \psi_{x, u} d \mu_{x}(u)
$$

is a decomposition of $\operatorname{Ind}_{K}^{G} \pi_{x}$ into irreducibles;

(iii) every decomposition of $\operatorname{Ind}_{K}^{G} \pi_{x}$ into irreducibles arises in the manner described in (ii).

ProOF. By the hypotheses on the sequence $1 \rightarrow K \rightarrow G \rightarrow Q \rightarrow 1$, the system $\left\{\gamma_{s}, \nu(s, t) ; s, t \in Q\right\}$ introduced at the start of $\S 5$ satisfies Assumption 3.1; with the notation already in use, the desired decomposition of $\rho^{K}$ is the one with diagonal subalgebra $\mathscr{D}$. The assertions (ii) and (iii) follow from the fact (Proposition 5.2) that the natural map, say $\varepsilon_{x}$, from $\operatorname{Ind}_{K}^{G_{x}} \rho_{x}\left(G_{x}\right)^{\prime}$ to $\operatorname{Ind}_{K}^{G} \pi_{x}(G)^{\prime}$, is an isomorphism, and the observation that if $\psi_{x}=\operatorname{Ind}_{K}^{G_{x}} \rho_{x}=\int_{U}^{\oplus} \psi_{x, u} d \mu_{x}(u)$ is a decompositon of $\psi_{x}$ with diagonal algebra $\mathscr{E}$, then the diagonal subalgebra for the decompositon $\hat{\rho}_{x}=\operatorname{Ind}_{G_{x}}^{G} \psi_{x}=\int_{U_{x}}^{\oplus} \operatorname{Ind}_{G_{x}}^{G} \psi_{x, u} d \mu_{x}(u)$ is $\varepsilon_{x}(\mathscr{E})$.

We note that in the case $K$ is type $I$ and $Q$ is arbitrary, it follows from [11, Theorem 8.1] that irreducible representations of $G_{x}$ restricting to a multiple of $\rho_{x}$ (such as $\psi_{x}$ ) induce to irreducible representations of $G_{x}$, establishing (more than) half the result. In the other direction, if $K$ is type I and $Q$ discrete, it follows from [12] that each irreducible decompositon of $\hat{\rho}_{x}$ is induced from an irreducible decomposition of $\psi_{x}$. Note, however, that it suffices to use representations induced from genuine subgroups of $G$ rather than being obliged to use also representations induced from representations (cocycles) of the "virtual subgroups" of $G$ arising from the action of $G$ on $\hat{K}$, as one might expect from the analysis in [12] of the irreducibles of $G$.

We should also point out that any generalization of Theorem 5.3 to nondiscrete groups $Q$ will have to contend with the possibility that the groups $Q_{x}$ need not, in general, be closed. The author believes that if the $Q_{x}$ are (almost) all closed, Theorem 5.3 should remain true, but no complete proof exists.

Also, we note that the cocycles $c_{x}$ appearing in Lemma 4.2 are precisely the Mackey obstruction attached to the representation $\rho_{x}$ of $K$-this follows from the observation that $\rho_{x} \circ \gamma_{s} \stackrel{\sim}{\sim} \rho_{x}$ if and only if $\pi_{x} \circ \alpha_{s} \stackrel{u}{\sim} \pi_{x}$, and that if $\operatorname{Ad} v_{s} \circ \pi_{x}=\pi_{x} \circ \alpha_{s}$, then $\operatorname{Ad} w_{s} \circ \rho_{x}=\rho_{x} \circ \gamma_{s}$ where $w_{s}=\pi_{x}\left(u_{s}^{*}\right) v_{s}$; in addition

$$
\begin{aligned}
w_{s} w_{t} & =\pi_{x}\left(u_{s}^{*}\right) \pi_{x}\left(\alpha_{s}\left(u_{t}^{*}\right)\right) v_{s} v_{t} \\
& =c_{x}(s, t) \pi_{x}\left(\gamma_{s}\left(u_{t}^{*}\right) u_{s}^{*} \omega(s, t)\right) v_{s t} \\
& =c_{x}(s, t) \rho_{x}(\nu(s, t)) w_{s t},
\end{aligned}
$$

so the obstruction to extend $\left.\rho_{x}\right|_{K}$ to a representation of $G_{x}$ is given by $c_{x} \in$ $Z^{2}\left(G_{x} / K, \mathbf{T}\right)$. 
Finally, we note that under the hypothesis of Theorem 5.3, the equivalence relation $(\mathscr{R}, \mu)$ is hyperfinite by [19] and [3], so that $H_{\mu}^{2}(\mathscr{R}, \mathbf{T})=0$. In addition, if $H$ is amenable, $\mathscr{R} \times{ }_{s} H$ is also amenable, (as a groupoid), so that so also are $\mathscr{R} \vee Q$ and $\mathscr{S}=(r, s)(\mathscr{R} \vee Q)$, the equivalence relation generated by $\mathscr{R}$ and the action of $H$. The analysis of [16, §2] now yields a homomorphism from $\mathscr{S} \rightarrow \mathscr{R} \vee Q$, and a decomposition $\mathscr{R} \vee Q=\mathscr{Q} \times s \mathscr{S}$ where $\mathscr{Q}$ denotes the Borel functor $x \in X \rightarrow \mathscr{Q}_{x}=Q_{x}$ with the natural action of $\mathscr{S}$. The description of $H_{\mu}^{2}(\mathscr{R} \vee Q)$ approached in $\S 2$ may now be completed via the exact sequence

$$
0 \rightarrow H_{\mu}^{1}\left(\mathscr{P} ; H_{\mu}^{1}(\mathscr{Q}, A)\right) \rightarrow H_{\mu}^{2}(\mathscr{R} \vee Q ; A) \rightarrow H_{\mu}^{2}(\mathscr{Q}, A) \rightarrow 0 .
$$

We turn now to some examples. Since discrete groups are never type I unless they have an abelian subgroup of finite index, and since being type I for a nontrivial multiplier is even more rare, we restrict attention to the case where $Q$ is abelian.

Note that if $0 \rightarrow A \rightarrow G \rightarrow Q \rightarrow 0$ is exact, with $A$ abelian, and $\theta$ is the multiplier associated to the extension, then the Mackey multiplier $c_{x}$ associated to the character $x \in \hat{A}$ is just

$$
c_{x}(s, t)=\left\langle x^{-1}, \theta(s, t)\right\rangle \quad \text { on } Q_{x} \times Q_{x} .
$$

If $c_{x}$ is trivial, the corresponding representation $\psi_{x}=\operatorname{Ind}_{A}^{G_{x}} x$ of $G_{x}$ has commutant which is isomorphic to $\lambda\left(Q_{x}\right)^{\prime}$, and $\psi_{x}$ admits a decomposition

$$
\psi_{x}=\int_{\tilde{G}_{x}}^{\oplus} p d_{x} p
$$

where $\tilde{G}_{x}$ denotes the group of all characters of $G_{x}$ which extend $x$ on $A$ (which is isomorphic to $\left.\hat{Q}_{x}\right)$ and $d_{x} p$ denotes normalized Haar measure on $\left(\hat{Q}_{x}\right.$ transferred to) $\tilde{G}_{x}$. Note that $P=\dot{U} \tilde{G}_{x}$ carries a standard Borel structure for which the natural action of $G$ by conjugation is Borel, using the results of [16]; in this case, one may also check that $x \rightarrow d_{x} p$ provides a Borel field of measure on $\tilde{G}_{x}$.

We know from the previous results of this section that

$$
\int_{X}^{\oplus} \int_{\tilde{G}_{x}}^{\oplus} \operatorname{Ind}_{G_{x}}^{G} p d_{x} p d \mu(x)
$$

provides a decomposition of $\rho^{G}$ with Cartan diagonal algebra. We will need a concrete realization of this fact, as follows. Let $\mathscr{S}$ denote the equivalence relation on $\dot{U} \tilde{G}_{x}$ coming from the action of $G$; for each $(p, q) \in \mathscr{S}$, we may choose $g=q_{p, q} \in G$ with $p \circ \operatorname{Ad} g_{p, q}=q$. Note

$$
g_{p, q} g_{q, r}=h(p, q, r) g_{p, r} \quad \text { for some } h(p, q, r) \in G_{p \mid A}
$$

and that $(p, q, r) \rightarrow h(p, q, r)$ is a 2 -cycle on $\mathscr{S}$ with values in $\left\{G_{x}: x \in X\right\}$. Since $\mathscr{S}$ is hyperfinite, by [3], $h$ is a coboundary and we may assume that $g_{p, q} g_{q, r}=q_{p, r}$ 
on $\mathscr{S}^{(2)}$. Let $L_{p}^{2}(G)$ denote the space of the representation $\operatorname{Ind}_{G_{x}}^{G} p=\hat{p}$, and note that for $\xi \in L_{q}^{2}(G)$ and $h \in G_{q \mid A}$

$$
\begin{aligned}
\xi\left(g_{q, p} h g g_{q, p}^{-1}\right) & =\xi\left(g_{q, p} h g_{q, p}^{-1} g_{q, p} g g_{q, p}^{-1}\right) \\
& =q \circ \operatorname{Ad} g_{q, p}(h) \xi\left(g_{q, p} g g_{q, p}^{-1}\right) \\
& =p(h) \xi\left(g_{q, p} g g_{q, p}^{-1}\right),
\end{aligned}
$$

so that $\left(U_{p, q} \xi\right)(g)=\xi\left(g_{q, p} g g_{q, p}^{-1}\right)$ defines a unitary from $L_{q}^{2}(G)$ to $L_{p}^{2}(G)$. Also

$$
\left(U_{p, q} \hat{q}(g) U_{p, q}^{*} \xi\right)\left(g^{\prime}\right)=\xi\left(g^{\prime} g_{q, p}^{-1} g g_{q, p}\right)=\left(\hat{p}\left(g_{q, p}^{-1} g g_{q, p}\right) \xi\right)\left(g^{\prime}\right)
$$

so that $\hat{p}\left(g_{q, p}\right) U_{p, q}$ is an intertwiner between $\hat{q}$ and $\hat{p}$. In addition, if $V_{p}: L_{p}^{2}(G) \rightarrow$ $l^{2}\left(r^{-1}(p)\right)$ is defined by $\left(V_{p} \xi\right)(r)=\xi\left(g_{p, r}\right)$, we have

$$
\begin{aligned}
\left(V_{p} \hat{p}\left(g_{q, p}\right) U_{p, q} V_{q}^{*} \xi\right)(r) & =\left(U_{p, q} V_{q}^{*} \xi\right)\left(g_{p, r} g_{q, p}\right)=\left(V_{q}^{*} \xi\right)\left(g_{q, p} g_{p, r}\right) \\
& =\left(V_{q}^{*} \xi\right)\left(g_{q, r}\right)=\xi(r)=(L(p, q) \xi)(r)
\end{aligned}
$$

Thus the representations $\rho_{p}=\operatorname{Ad} V_{p} \circ \hat{p}$ have the property that $\operatorname{Ad} L(p, q) \circ \rho_{q}=$ $\rho_{q}$, where $L$ is the left regular representation of the equivalence relation $\mathscr{S}$. It now follows from [13] the diagonal algebra of $\int_{X}^{\oplus} \int_{\tilde{G}_{x}} \rho_{p} d_{x} p d \mu(x)$ is a Cartan subalgebra.

We now compute the data necessary for the Plancherel formula of [15], using the representations $\rho_{p} ; 1_{q}$ denotes the characteristic function of $q \in \dot{U} \tilde{G}_{x}$, viewed as an element of $l^{2}\left(r^{-1}(p)\right)$ for any $p$ with $(p, q) \in \mathscr{S}$. Observe that in our situation, the groups $G_{x}$ are normal in $G$ and hence constant, $G_{x}=G_{0}$, at least on ergodic components of the action of $G$ on $X$. Also, we have $g_{p, r} g_{p, s}=$ $h_{p}(r, s) g_{p, r g_{p, o}}$ for some $h_{p}(r, s) \in G_{p \mid A}=G_{0}$; in fact $h_{p}$ may be viewed as the 2-cycle naturally attached to the extension

$$
1 \rightarrow G_{p \mid A} \rightarrow G \rightarrow G / G_{p \mid A} \rightarrow 1 .
$$

for each $p$. We compute

$$
\left(\rho_{p}(g) 1_{q}\right)(r)=\left(V_{p} \hat{p}(g) V_{p}^{*} 1_{q}\right)(r)=\left(V_{p}^{*} 1_{q}\right)\left(g_{p, r} g\right) .
$$

Thus, when $g=h \in G_{0}$, we have

$$
\left(\rho_{p}(h) 1_{q}\right)(r)=p\left(g_{p, r} h g_{p, r}^{-1}\right) 1_{q}(r)=\left(q(h) 1_{q}\right)(r),
$$

and

$$
\begin{aligned}
\left(\rho_{p}\left(g_{p, s}\right) 1_{q}\right)(r) & =\left(V_{p}^{*} 1_{q}\right)\left(h_{p}(r, s) g_{p, r g_{p}, s}\right) \\
& =p\left(h_{p}(r, s)\right) 1_{q}\left(r g_{p, s}\right) . \\
& =p\left(h_{p}\left(q g_{s, p}, s\right)\right) 1_{q g_{s, p}}(r)
\end{aligned}
$$


Thus for $\varsigma \in l^{1}(G) \cap l^{2}(G)$. We have

$$
\begin{aligned}
\rho_{p}(\zeta) 1_{q} & =\sum_{s \sim q} p\left(h_{p}\left(q g_{s, p}, s\right)\right)\left(\sum_{h \in G_{0}} \zeta\left(h g_{p, s}\right) q g_{s, p}(h)\right) 1_{q g_{s, p}} \\
& =\sum_{s \sim q} p\left(h_{p}\left(q g_{s, p}, s\right)\right)(p, s \zeta)^{\wedge}(q) 1_{q g_{s, p}}
\end{aligned}
$$

where $(p, s \zeta)^{\wedge}(q)$ is, by definiton, $\sum_{h \in G_{0}} \zeta\left(g_{p, s} h\right) q(h)$, since

$$
\begin{aligned}
\sum_{h \in G_{0}} \varsigma\left(h g_{p, s}\right) q g_{s, p}(h) & =\sum_{h \in G_{0}} \varsigma\left(h g_{p, s}\right) q\left(g_{s, p} h g_{p, s}\right) \\
& =\sum_{h \in G_{0}} \varsigma\left(g_{p, s} h\right) q(h) .
\end{aligned}
$$

Thus $\left\|\rho_{p}(\zeta) 1_{q}\right\|^{2}=\sum_{g \sim q}\left|(p, s \zeta)^{\wedge}(q)\right|^{2}$, since the vectors $1_{q g_{s, p}}$ are orthogonal as $s$ varies, and $p$ is a character. Note that in fact this quantity is independent of $p$ with $(p, q) \in \mathscr{S}$, and hence

$$
\left\|\rho_{p}(\varsigma) 1_{q}\right\|^{2}=\sum_{s \sim q}\left|(q, s \zeta)^{\wedge}(q)\right|^{2}
$$

and the Plancherel formula of [15] for $G$ takes the form

$$
\|\zeta\|_{2}^{2}=\int_{X} \int_{\tilde{G}_{x}} \sum_{(s, q) \in \mathscr{S}}\left|(q, s \zeta)^{\wedge}(q)\right|^{2} d_{x} q d \mu(x),
$$

for $\zeta \in l^{1}(G) \cap l^{2}(G)$. To the author's knowledge, formulae of this type have not appeared before.

We note finally that in attempting to apply the analysis of $\S \S 2,3,4$ to extensions of the type

$$
1 \rightarrow G \rightarrow G_{1} \rightarrow Q_{1} \rightarrow 1
$$

where $Q_{1}$ is discrete abelian, and $G$ is of the type already considered, that is, an extension $1 \rightarrow A \rightarrow G \rightarrow Q \rightarrow 1$ with $A$ and $Q$ discrete abelian, one encounters the problem that the decomposition of $\rho^{G}$ already displayed might not have suitable invariance properties under the action of $G_{1}$-certainly if $G_{1}$ leaves $A$ invariant, it will conjugate the isotropy groups $G_{x}$ appropriately, and the analysis carries through. Details are left to the reader. However, in other situations, we must modify the action of $G_{1}$ by a cocycle which is very difficult to find explicitly, and the formalism becomes much more difficult to control. 


\section{References}

[1] A. Connes, Sur la theorie non-commutative de l'integration (Springer Lecture Notes in Math., No. 725).

[2] A. Connes, 'Classification of injective factors: Cases $\mathrm{II}_{1}, \mathrm{II}_{\infty}$ and $\mathrm{III}_{\lambda}, \lambda \neq 1$, Ann. of Math. (2) 104 (1976), 73-115.

[3] A. Connes, J. Feldman and B. Weiss, 'An amenable equivalence relation is generated by a single transformation', Ergodic Theory Dynamical Systems 1 (1981), 431-450.

[4] J. Dixmier, 'Sur la représentation réguliere d'une groupe localement compact', Ann. Sci. Ecole Norm. Sup. 2 (1969), 423-436.

[5] J. Dixmier, Les $C^{*}$-algèbres et leurs représentations (Gauthier-Villars, 2eme edition, Paris, 1968).

[6] J. Feldman and C. C. Moore, 'Ergodic equivalence relations, cohomology, and von Neumann Algebra', I and II, Trans. Amer. Math. Soc. 274 (1977), 289-324 and 325-359.

[7] P. Green, 'The local structure of twisted covariance algebras', Acta Math. 140 (1978), 191-250.

[8] U. Haagerup, 'Standard forms of von Neumann algebras', Math. Scand. 37 (1975), 271283.

[9] P. Hahn, 'Reconstruction of a factor from measures on Takesaki's unitary equivalence relation', J. Funct. Anal. 31 (1979), 263-271.

[10] V. F. R. Jones and M. Takesaki, 'Actions of compact abelian groups on semifinite injective factors', Acta Math. 153 (1984), 213-258.

[11] G. W. Mackey, 'Unitary representations of group extensions I', Acta Math. 99 (1958), 265-311.

[12] A. Ramsay, 'Non-transitive quasi-orbits in Mackey's analysis of group extensions', Acta Math. 137 (1976), 17-48.

[13] C. Sutherland, 'Maximal abelian subalgebras of von Neumann algebras, and representations of equivalence relations', Trans. Amer. Math. Soc. 280 (1983), 321-336.

[14] C. Sutherland, 'Cohomology and extensions of von Neumann algebras II', Publ. Res. Inst. Math. Sci. 16 (1980), 135-174.

[15] C. Sutherland, 'Cartan Subalgebras, Transverse measures and non-type I Plancherel formulae', J. Funct. Anal. 60 (1985), 281-308.

[16] C. Sutherland, 'A Borel parametrization of Polish groups', Publ. Res. Inst. Math. Sci. 21 (1985), 1067-1086.

[17] C. Sutherland and M. Takesaki, 'Actions of discrete amenable groups and groupoids on semifinite injective von Neumann algebra', Publ. Res. Inst. Math. Sci. 21 (1985), 10871120.

[18] M. Takesaki, 'On the unitary equivalence relation among components of decompositions of representations of involutive Banach algebras, and the associated diagonal algebras', Tohuku Math. J. 15 (1963), 365-393.

[19] R. Zimmer, 'Hyperfinite factors and amenable ergodic actions', Invent. Math. 41 (1977), 23-31.

\section{Mathematics Department}

The University of New South Wales

P.O. Box 1

Kensington, N.S.W. 2033

Australia 